\title{
ROMA E IL SUO IMPERO PREGLOBALE. UNA LEZIONE PER LA POSTMODERNITÀ*
}

\author{
Orazio Licandro \\ Dipartimento di Scienze Humanistiche, Università degli \\ Studi di Catania, Itália \\ oralicandro@gmail.com
}

\begin{abstract}
RIASSUNTO: Il presente articolo adotta un approccio storico al sistema di governo dell'Impero romano e al modo in cui i problemi che coinvolgono il sistema di governo e i cittadini sono stati risolti. Il lavoro descrive anche le relazioni instaurate con le nazioni in tutto il bacino del Mediterraneo, l'impatto economico, poli-tico e sociale generato dalle politiche e guerre imperialiste per conquistare nuovi territori, il pensiero politico e giudiziario, la formazione di un modello di Il "costituzionalismo" caratteristi-co del mondo romano, il rapporto con la scienza giuridica dell'epoca, nonché alcuni istituti di diritto civile e diritto urba-no. In considerazione di ciò, il lavoro mira a offrire una rifles-sione in quanto la legge romana, cercando di risolvere i pro-blemi sociali, economici e politici dell'antichità, può servire come ispirazione per i problemi della postmodernità. La ricer-ca è bibliografica, esplorativa, documentaria e descrittiva. Alla fine dell'indagine, si è concluso che, tornando indietro nel tempo, è possibile capire se e come problemi simili di urgenza contemporanea siano stati affrontati e affrontati in questa straordinaria esperienza legale la cui eredità oggi è preziosa. In tal modo, molte delle soluzioni trovate per quel tempo pos-sono servire da modello per la legge, per la scienza giuridica e per il mondo postmoderno.
\end{abstract}

PAROLE CHIAVE: Diritto Romano. Globalizzazione. Postmodernità.

\section{Rome and its pre-global empire A lesson for postmodernity}

ABSTRACT: The present article takes a historical approach to the system of go-vernment of the Roman Empire and how problems that involved the system of government and citizens were solved. The work also descri-bes the relations established with nations across the Mediterranean basin, the economic, political and social impact generated by imperia-list policies and wars to conquer new territories, political and judicial thinking, the formation of a model of "constitutionalism" characteri-stic of the Roman world, the relationship with the Legal Science of the time, as well as some institutes of civil law and urban law. In view of this, the work aims to offer a reflection as Roman law, seeking to sol-ve social, economic and political problems of antiquity, may well serve as inspiration for the problems of postmodernity. The research is bi-bliographic, exploratory, documentary and descriptive. At the end of the investigation, it was concluded that, going back in time, it is pos-sible to understand whether and how similar problems of urgent con-temporaneity were taken and approached in this extraordinary legal experience whose legacy today is precious. In such a way, many of the solutions found for that time can serve as a model for the post-moderns Law, Legal Science and world.

KEYWORDS: Roman Law. Globalization. Post-modernity.

\footnotetext{
* Si tratta del testo modificato e approfondito della relazione dal titolo "Sovranità, cittadinanza, persona e territorio in un impero preglobale. L'esperienza augustea", tenuta al Convegno "Le sovranità nell'era della post globalizzazione", Foggia, 1 marzo 2019.
} 


\section{Roma e seu império pré-global. Uma lição para a pós-modernidade}

RESUMO: O presente artigo faz uma abordagem histórica do sistema de governo do Império Romano e de como problemas que envolviam o sistema de governo e cidadãos eram resolvidos. O trabalho descreve ainda as relações estabelecidas com nações de toda a bacia do Mediterrâneo, o impacto econômico, político e social gerado em razão das políticas imperialistas e das guerras de conquista de novos territórios, o pensamento político e judicial, a formação de um modelo de "constitucionalismo" característico do mundo romano, a relação com a Ciência Jurídica da época, bem como alguns institutos do direito civil e do direito urbanístico. Diante disso, o trabalho visa oferecer uma reflexão como o direito romano, buscando equacionar problemas sociais, econômicos e políticos da antiguidade, pode muito bem servir de inspiração para os problemas da pós-modernidade. A pesquisa é bibliográfica, exploratória, documental e descritiva. Ao cabo da investigação, concluiu-se que, voltando no tempo, é possível entender se e como problemas semelhantes da contemporaneidade urgente foram tomados e abordados nessa extraordinária experiência jurídica cujo legado hoje é precioso. De tal forma, muitas das soluções encontradas para aquela época podem servir de modelo para o Direito, para a Ciência Jurídica e para o mundo pós-modernos.

PALAVRAS-CHAVE: Direito Romano. Globalização. Pós-modernidade.

\section{Premessa}

È il 14 d.C., a Nola muore Augusto, le celebrazioni dei funerali durano un mese, Roma e l'Italia sono in preda a una parossistica commozione, corre un'isteria collettiva, il senato è persino indotto a decretare un freno agli eccessi ${ }^{1}$. Il princeps, il primo cittadino, il pacificatore, il migliore per charisma, virtù, meriti, aveva concluso la sua vicenda umana, soprattutto una straordinaria vicenda politica e istituzionale, e aveva lasciato una Roma assai diversa. Un vastissimo impero geograficamente esteso su tre continenti, che qualche secolo dopo nella sua successiva massima estensione sarebbe andato da Dura Europos in Mesopotamia, strategico snodo commerciale sull'Eufrate, al Vallo di Adriano in Britannia, dalla Gallia e dalla Spagna alle propaggini meridionali dei deserti nordafricani, si pensi anche alle coorti di stanza a Syene (Assuan) o al fortilizio a Qasr Ibrim, o al distaccamento romano sulle isole Farasan (oggi territorio dell'Arabia Saudita) per il controllo del Mar Rosso. Nelle sue Res Gestae, Augusto ricordava gli esiti dei tre censimenti dei cives Romani (28 a.C. $=4.073 .000 ; 18$ a.C. $=4.233 .000 ; 14$ d.C. $=4.937 .000$ ), ma la popolazione complessiva dell'impero consisteva di circa 54 milioni di abitanti.

Ancora. Un impero segnato da relazioni fittissime asimmetriche con popoli e nazioni dell'intero bacino del Mediterraneo, attraversato da una miriade di culti e idiomi, ma con due grandi aree latinofona e grecofona, culture ed economie diverse e un ricchissimo e variegato pluralismo normativo.

Questa realtà era il risultato di 40 anni di governo e di riforme, di una transizione lunga e difficile e della ricostruzione istituzionale, politica, economica e sociale della res publica. Un gigantesco disegno di rifondazione perseguito con sagacia e abilità da Augusto, chiusa la stagione terribile delle guerre civili, che avevano disintegrato il tessuto sociale ed economico e

1 Tac. ann. 1, 8, 5: Remisit Caesar adroganti moderatione, populumque edicto monuit ne, ut quondam nimiis studiis funus divi Iulii turbassent, ita Augustum in foro potius quam in campo Martis, sede destinata, cremari vellent. 
sovvertito radicalmente un mondo con i suoi valori (si pensi soltanto al catalogo delle nefandezze delle proscrizioni) $)^{2}$.

Il collasso della Roma repubblicana aveva però radici più antiche e profonde nelle potenti trasformazioni economiche che avevano frantumato la politica e le sue istituzioni e travolto gli antichi equilibri di potere fondati su consolidati assetti sociali. Il grande latifondo schiavile, con le villae vere e proprie strutture economiche produttive più che di lusso finalizzato all' otium, con le sue nuove forme di sfruttamento e produzione massicce e intensive, aveva prodotto la polverizzazione di quel ceto medio e piccolo di agricoltori/cittadini/soldati, alla base del successo di Roma. D'altro canto, un contraccolpo potente, diretta conseguenza delle politiche imperialiste e delle guerre di conquista di nuovi territori, fu la formazione di enormi sacche di nullatenenti inurbati preda di demagoghi e populisti e la degenerazione del ricorso alla violenza come metodo di lotta politica, praticata da bande anche armate. Sulla scena politica, peraltro, da tempo si assisteva all'irruzione e al consolidamento di un ceto mercantile e affaristico aggressivo e spregiudicato, capace di impiegare ingenti patrimoni per scalare il potere politico, insomma, portatore di un nuovo concetto di ricchezza che dissolveva «gli arcaismi catoniani» ${ }^{3}$. I più dinamici e marcati tratti mercantili dell'economia avevano, del resto, già esercitato una forte influenza pure sul quadro istituzionale, con la comparsa, intorno alla metà del III secolo a.C., della figura del praetor peregrinus, un nuovo magistrato con il compito di amministrare la giustizia tra stranieri o tra cittadini e stranieri su questioni, diremmo oggi, di 'diritto commerciale' 4 .

Unificazione dello spazio Mediterraneo dall'accresciuta sicurezza e perciò pieno di vele, produzioni e mercati lontani, ricchezza pecuniaria che, dilatando «artificialmente sia la disponibilità dei fattori di produzione - lavoro, terra e capitali - sia la domanda di mercato $»^{5}$, finiva per marcare un segno anomalo alla tradizionale nobiltà romana diffidente verso l'imprenditorialità su vasta scala ${ }^{6}$, relazione tra commerci e fiscalità virtuosa ${ }^{7}$, tutto ciò spingeva Roma verso $i$ vantaggi di una 'mondializzazione' che nei primi due secoli dell'era cristiana si sarebbero effettivamente materializzati ${ }^{8}$.

Il richiamo di questo quadro generale forse con eccessivo schematismo, era necessario per fissare, a scanso di equivoci, una cornice all'intervento: evitare di pensare che le profonde trasformazioni in atto nel nostro tempo, ora definito della globalizzazione o della postglobalizzazione, ora della postmodernità, siano inedite. Le grandi trasformazioni generano sempre transizioni difficili, complesse, delicate; investono temi cardinali, come quelli della sovranità, cittadinanza, identità, migrazioni, costanti perenni tanto delle esperienze statuali moderne quanto di quelle antiche.

Ora, che nell'antichità il segno delle relazioni dei grandi complessi di potere, regni o imperi, fosse diverso da quello delle attuali è abbastanza ovvio, che le relazioni fossero certamente

2 Per un quadro della sterminata letteratura rimando a O. LICANDRO, Augusto e la res publica imperiale. Studi epigrafici e papirologici, Torino 2018.

L. CAPOGROSSI COLOGNESI, Padroni e contadini nell'Italia repubblicana, Roma 2012, 163.

4 Un quadro organico in P. CERAMI - A. PETRUCCI, Diritto commerciale romano. Profilo storico, Torino 2010.

L. CAPOGRossi Colognesi, Padroni e contadini, cit., 164.

6 Per un quadro d'insieme vedi O LICANDRO, Dalla lex Claudia de quaestu senatorum alle leges repetundarum ovvero del conflitto di interessi nell'antica Roma, in Fides Humanitas Ius. Studii in onore di L. Labruna, IV, Napoli 2007, 2815 ss.

7 A. SCHIAVOnE, La storia spezzata. Roma antica e Occidente moderno, Roma-Bari 2002, 205.

8 Viene considerato un deciso fautore Plin. nat. hist. 27, 1, 2-3, proponendo addirittura un nesso causale tra dèi, natura, pace e dominio romano. Sul tema, oltre ai classici M. RosTOVZEV, Storia economica e sociale dell'impero romano, Firenze 1980; e F. DE MARTINO, Storia economica di Roma antica, II, Firenze 1979; nuovi spunti in L. CAPOGROSSI COLOGNESI, A Provocation, in Rivista di Storia Economica 25 (2009) 421 ss.; E. Lo CASCIO, L'economia imperiale e la svolta augustea, in AA.VV., Augusto e la costruzione del principato. Atti del Convegno (Roma, 4-5 dicembre 2014), Roma 2017, 327 ss. 
molteplici ma non globali, come possiamo intenderle oggi, altrettanto evidente. Eppure, per l'impero romano, come già osservato da Carl Schmitt, le cose stettero diversamente rispetto ad altre esperienze statuali dell'antichità ${ }^{9}$.

Naturalmente, non vi è alcuna pulsione di Aktualisierung, né alcun tentativo di accostare un'esperienza storica concreta, dunque cose lontanissime e diversissime, per proporre ricette adeguate alla complessità dei nostri tempi; semmai la suggestione di ragionare appunto sulla perennità di alcune grandi questioni, in connessione con autorevoli visioni campeggianti in altre discipline scientifiche, e così profittandone, soprattutto nella temperie che stiamo attraversando in cui la scienza giuridica, forse più che nel passato, schiacciata tra fondamentalismi di natura diversa - teologie, tecnocrazia e tecnologia - deve affrontare la questione stessa della sua esistenza.

\section{1. 'Costituzionalismo' a Roma}

Nell'assenza di una carta costituzionale, persino di un concetto maturo, consapevole di costituzione, che è prodotto moderno della Storia e degli Stati nazionali, soltanto dal III-II secolo a.C., sulla base della documentazione sopravvissuta, sappiamo che a Roma il pensiero politico e giuspubblicistico aveva cominciato a riflettere su un frammentario nucleo normativo, composto di norme scritte (poche), consuetudini, prassi, convenzioni costituzionali che definivano complessivamente un assetto istituzionale e le relazioni tra i vari organi 'costituzionali'. Non una costituzione, ma un nucleo normativo duttile e cangiante a seconda delle fasi, degli eventi, dei mutamenti degli equilibri politici e in tal modo via via stratificatosi. Lo stesso termine constitutio, nonostante l'assonanza e l'invalsa utilizzazione del termine, è del tutto fuorviante. I Romani usavano, infatti, altre espressioni: ad esempio status civitatis, status rei publicae, ma soprattutto forma rei publicae. Espressione, questa, non indicativa né di una precisa forma di Stato cioè una 'Repubblica', né di una precisa forma di governo (appunto repubblicana), ma di una comunità res populi - fondata sulla libertas (dunque, cives liberi e non sudditi), governata attraverso una serie di organi (magistrati, assemblee popolari e senato) concorrenti alle decisioni fondamentali, in un assetto istituzionale incompatibile con il regnum: potremmo dire che la vera Grundnorm repubblicana era l'adfectatio regni $=$ aspirazione al regnum, alla tirannia ${ }^{10}$.

Quella romana, in effetti, fu esperienza assai peculiare e profondamente diversa sia dalle monarchie ellenistiche e orientali dispotiche sia dall'esperienza squisitamente greca della polis. Elementi di straordinaria modernità la connotavano: Polibio, ad esempio, raffinato osservatore interno al circolo degli Scipioni nel II secolo a.C., ne apprezzava l'originale sistema di governo misto ${ }^{11}$, un impianto di pensiero che un secolo dopo veniva rivisitato da Cicerone nel De re

\footnotetext{
9 C. SCHMITT, Il nomos della terra nel diritto internazionale dello «jus publicum europaeum», Milano 1991. Ma sul tema con approcci diversi A. BANCALARI MOLINA, Orbe Romano e Imperio Global. La Romanización desde Augusto to Caracalla, Santiago 2007; R. Hingley, Globalizing Roman Culture. Diversity and Empire, London-New York 2005; R.B. HiTCHNER, The First Globalization. The Roman Empire and Its Legacy in the 21 st Century, Oxford 2007; O. VEYNE, L'Impero greco romano. Le radici del mondo globale, Milano 2007.

${ }^{10} \mathrm{Sul}$ tema si rinvia ai più recenti contributi di F. RUSSO, L'odium regni a Roma tra realtà politica e finzione storiografica, Pisa, 2015, passim; F. ZuCCOTTI, Sacramentum civitatis. Diritto costituzionale e ius sacrum nell'arcaico ordinamento giuridico romano, Milano 2016, 26 ss.

${ }^{11}$ Su Polibio si veda il recentissimo libro di G. ZECCHINI, Polibio. La solitudine dello storico, Roma 2018.
} 
publica, il suo trattato politico per eccellenza. Cicerone, oltre alla maggiore definizione dei diversi genera rei publicae ${ }^{12}$, fissava seccamente il nesso libertas e populus: nessuna libertas sarebbe stata possibile se non in una civitas in cui summa potestas est in populi (Cic. de re publ. 1, 31, 47).

Il senato certo, i magistrati certo, ma una res publica poteva dirsi tale in quanto res populi, e pertanto nel populus si sedimentava la relativa concezione di fonte di sovranità politica, nelle sue diverse forme legislativa, elettiva e giurisdizionale, e di cui ancora oggi parliamo, sebbene negli ultimi tempi declinata improvvidamente in populismo.

\section{Consensus universorum e Potentia. Potere e AuCtori- TAS. POPUluS E CENTRALITÀ DELLA LEX.}

«A Roma il consenso politico, nell'equilibrio contemperato tra le varie fasce sociali, era elemento di cui nessun imperatore avveduto, pur nel suo potere indiscusso e assoluto, poteva fare a meno. Il consenso istituiva un mutuo dialogo di potere ed era parte costitutiva di ogni politica del giusto mezzo. Alla lunga ignorarlo o non rinnovarlo in forma diffusa e bilanciata metteva necessariamente a repentaglio la vita» ${ }^{13}$. Tutto ciò era già valso per Cesare, e Augusto seppe trarne la lezione. Trovandosi a fare i conti con questo primo problema, decise di farlo rinunciando ai poteri triumvirali speciali e ripristinando il normale funzionamento degli organi repubblicani: senato e assemblee del popolo.

Sconfitti Antonio e Cleopatra, Augusto restava il dominatore incontrastato della scena politica. Godeva di un consenso generale, era il pacificatore, colui che aveva scacciato il caos, riportato ordine e normalità. Godeva di un consenso generale ed era potens, come lui stesso diceva in quella testimonianza colma di ebbrezza di se stesso ${ }^{14}$. Già prima la coniuratio l'aveva consacrato come guida militare, e avrebbe potuto continuare a far leva su questa situazione nella relazione con il populus. Insomma, privo di alcun limite dinanzi a sé, Augusto avrebbe potuto imboccare una strada assai diversa dalla restitutio rei publicae, come tutti i presupposti lasciavano intendere, a favore di una forma spiccatamente monarchica. Pur in condizioni di potenza, aveva la necessità di trasformare il consenso in potere. Avrebbe potuto costruire un rapporto inedito con il popolo, superando aristocrazie, élites, facendo leva su spinte oggi, diremmo, populiste, e cancellare del tutto le istituzioni repubblicane; e, invece, si mosse in una direzione contraria, rinunciando alla potentia, dietro la consapevolezza della delicatezza della fase e della complessità di un impero così vasto, con culture e identità tanto diverse e lontane.

La potentia, infatti, nel lessico e nell'ideologia repubblicani assumeva una connotazione negativa quale situazione di fatto fuori della legalità. Bisognava al contrario intraprendere una direzione molto più rassicurante, quindi scelse l'auctoritas, figura antica, ben conosciuta, del diritto privato e pubblico (dell'auctoritas godevano pure i giuristi), esprimente su quest'ultimo

${ }^{12} \mathrm{Su}$ cui vedi M. MICELI, 'Governo misto', quartum genus rei publicae e separazione dei poteri, in AA.VV., Tradizione romanistica e Costituzione (dir. L. Labruna - a cura di M.P. Baccari e C. Cascione), I.1, Napoli, 2006, 659 ss.; P. CERAMI - M. Miceli, Storicità del diritto. Strutture costituzionali, Fonti, Codici. Prospettive romane e moderne, Torino 2018, 64 ss.; cfr. O. LiCANDRO, Cicerone alla corte di Giustiniano. "Dialogo sulla scienza politica” (Vat. gr. 1298). Concezioni e dibattito sulle formae rei publicae nell'età dell'assolutismo imperiale, Roma, 2017, passim.

13 O.D. CORdovanA, I Severi, in AA.VV., Roma universalis. L'impero e la dinastia venuta dall'Africa, Milano 2018, 36 (catalogo della mostra).

${ }^{14}$ Sulla nuova integrazione di $R G D A 34,1-3$ e per il dibattito storiografico dispiegatosi mi limito a rimandare a $\mathrm{O}$. LICANDRO, Augusto e la res publica imperiale, cit., passim. 
piano il prestigio, il charisma, l'autorevolezza di un cittadino che per sue virtù e meriti era riconosciuto tale da tutti e che pertanto si poneva al di sopra di ogni cosa: insomma, il migliore dei cittadini.

Scelta la direzione, bisognava giungere sino alla fine e riaffermare un'idea di sovranità popolare che al tempo stesso si fondasse sulla ricerca di una rinnovata identità. La via fu quella di ancorarsi saldamente all'imperium populi Romani che, al di là della valenza egemonica sottesa, indicava nel populus Romanus il centro politico di legittimazione del potere. La scelta fu giocata con successo.

Anche nei secoli successivi, in un principato ormai dai tratti ben definiti e, certamente, monarchici, il popolo, con il suo atto fondamentale, cioè la lex, rimase la fonte di legittimazione del potere imperiale; è esplicito Gai. 1, 5, ma soprattutto:

\section{1, 4, 1 pr. (Ulp. 1 inst.): Quod principi placuit, legis habet vigorem: utpote cum lege regia, quae de imperio eius lata est, populus ei et in eum omne suum imperium et potestatem conferat.}

Non è un caso che Ulpiano, quando nell'età dei Severi si procedette alla teorizzazione del fondamento di legittimità della volontà normativa del principe, abbia considerato epilogo coerente ricondurla alla matrice popolare: era, infatti, mediante lex che il popolo delegava l'imperium al princeps (sebbene il populus fosse ben altra cosa del populus del I secolo a.C. - I secolo d.C.).

$$
* * *
$$

Non solo, conformemente al motivo propagandistico della restitutio rei publicae, restaurò il funzionamento di senato e assemblee popolari, ma puntò sulla centralità della legge facendone uno strumento fondamentale sia per produrre diritto sia per modificare la forma rei publicae. Se si vuole, potremmo scorgervi un approccio moderno, presente in alcune recenti sentenze della nostra Corte Costituzionale (nn. 148, 151, 198 del 2012), secondo la quale la salus rei publicae non ammette rimedi extracostituzionali, dato che ogni emergenza deve essere affrontata con i rimedi consentiti dall'ordinamento costituzionale.

Ad ogni modo, Augusto recuperava una concezione diffusa nel pensiero politico e giuridico tardorepubblicano e che possiamo leggere addirittura in un giovane Cicerone ${ }^{15}$, che interpretava la lex quale potente rimedio dei mali della res publica. La visione organicistica dello Stato romano e della lex come medicina di un corpo gravemente malato sarebbe stata uno dei motivi dominanti ampiamente documentati dai testi giuridici tardoimperiali sino alla produzione novellistica di Giustiniano. Ma, al di là di questa considerazione, il punto centrale della riflessione e dell'elaborazione della politica istituzionale augustea fu proprio la centralità della lex: si può senz'altro dire che, nell'orizzonte di un impero che aveva sconfitto l'opzione monarchica orientale, si stagliava con prepotenza la figura del legislatore.

Tutti i passaggi, gli aggiustamenti, i consolidamenti, le innovazioni, i rinnovi dei suoi poteri, che ne plasmarono nel complesso la posizione costituzionale, avvennero mediante leges. Altrettanto accadde per le riforme di diritto sostanziale o processuale (si pensi alla poderosa riforma sulla giurisdizione, con le leges Iuliae iudiciorum privatorum et publicorum). Importa poco quanto fosse sincero o studiato il rispetto che Augusto mostrasse verso questa fonte di produzione normativa, poco importa quanto riuscisse a orientare tutto: un fatto indiscutibile sul

15 Cic. de inv. $1,68$. 
piano formale, e la forma soprattutto nel 'costituzionalismo antico' è sostanza, è che attraverso la legge egli introdusse quei nuovi fundamenta nel corpo sfibrato dello Stato romano a cui si allude nella biografia svetoniana ${ }^{16}$.

Non a caso, Tito Livio (2.3.3-4), storico augusteo per eccellenza, nel racconto del passaggio dalla monarchia alla repubblica, fa pronunciare ai sostenitori del tiranno cacciato, Tarquinio il Superbo, parole di disprezzo verso la legge come qualcosa di 'sordo e inesorabile', e perciò il più bell'elogio della legge. Certo, si odono echi dell'antico dilemma tra Antigone e Creonte, ma la legge con la sua forma scritta in cui si versavano principi, norme, regole svolgeva una funzione di stabilità ${ }^{17}$ e, dunque, rassicurante per un mondo sprofondato nel caos di un secolo di guerre civili.

Persino nella ricostruzione di quell'identità frantumata, Augusto, mirando alla rivitalizzazione degli antichi mores maiorum, fece leva sulla lex (le leggi matrimoniali, quelle demografiche e sociali, ecc.), ridefinendo mediante essa i principi fondamentali della convivenza sociale.

Il princeps, il gubernator, il timoniere ciceroniano, il migliore per virtù e meriti, capace di condurre il governo di una res publica anche nei momenti più difficili, chiamava tutti a una renovatio etica riconoscendosi nella lex quale espressione della volontà popolare lo strumento per prescrivere l'ordinamento futuro.

\section{LA COSTRUZIONE DELL'IDENTITÀ}

Fu lungo questa prospettiva identitaria che Augusto dovette affrontare un altro nodo politico fondamentale, cioè il rinnovamento della classe dirigente, chiamando attorno alla sua visione di risanamento della res publica la migliore intellettualità, e rendendo quella visione patrimonio collettivo. Lo fece con metodo nella costruzione di una poderosa narrazione identitaria.

1) Razionalizzò quel fascio difficilmente districabile e anche contraddittorio sulle origini di Roma di racconti, tradizioni, miti greci: nacque, infatti, la grande letteratura nazionale con Virgilio, Livio, Orazio, Properzio, Tibullo, il grandissimo Ovidio ${ }^{18}$, finché non entrò, per ragioni ancora oggi ignote, in rotta di collisione con il principe.

2) Elaborò, con straordinaria abilità, una vera e propria, ancorché embrionale, teologia imperiale sulla propria persona declinata con una duplice modalità: cumulo delle cariche sacerdotali (per l'Occidente); presentazione di se stesso come uomo-dio inviato dalla provvidenza divina con la missione di portare sulla terra una nuova èra di pace (si veda l'emblematica iscrizione di Priene del 9 a.C. $)^{19}$, per incontrare e interpretare in senso originale le attese soteriologiche di un messianismo largamente serpeggiante nei territori orientali (si pensi soltanto a quello giudaico), e diffondendo, in quell'impero vastissimo segnato da innumerevoli culti, quello unitario del Genius Augusti. Diversità ma identità: un altro germe di una visione ecumenica o globale.

3) Rinnovò in profondità la classe dirigente. Non discriminò gli oppositori (esemplare il caso del giurista Labeone a cui offrì persino il consolato), e puntò decisamente sugli homines novi, l'ordine equestre, depositario di gran parte della ricchezza in circolazione. I cavalieri

16 Svet. Aug. 28, 1-4.

${ }^{17}$ Si trattò di una concezione talmente radicata da essere pienamente trasmessa alle esperienze legislative romano-germaniche; si veda persino il caso dell'Editto del re longobardo Rotari; Ed. Roth. cap. 386.

${ }^{18} \mathrm{Su}$ Ovidio, si legga il bel libro di F GHEDINI, Il poeta del mito. Ovidio e il suo tempo, Roma 2018; e i vari contributi raccolti nel catalogo della mostra AA.VV., Ovidio. Amori, miti e altre storie, Roma 2018.

19 SEG IV, 490 (= OGIS II, 458). 
costituivano il ceto sociale più dinamico e attivo nel sistema produttivo economico, negli investimenti, diventando così altro del semplice contraltare concorrente della vecchia aristocrazia terriera raccolta nell'ordine senatorio, ma un ceto strategico aderente al progetto augusteo per il rilancio dell'impero. Si potrebbe dire che l'abilità augustea realizzò una sorta di istituzionalizzazione, o canalizzazione, della ricchezza privata verso il fine generale, pubblico, della ricostruzione della res publica.

Non solo, ma saggiamente, Augusto mise in pratica una politica di integrazione delle élites locali in una visione generale di una loro comune partecipazione al potere e al disegno della casa imperiale. Il populus augusteo, naturalmente sotto il profilo concettuale non coincideva più con il populus di una città-stato. Il populus era costituito dall'insieme dei cives dislocati in tutto l'impero romano, di fatto ormai una federazione o, con metafora assai più suggestiva, una costellazione di poleis, di città, e di innumerevoli popoli o genti. Obiettivo reale era fermare o, addirittura, prevenire forze e spinte centrifughe e disgregatrici. La strategia adottata fu, dunque, quella dell'estensione della cittadinanza, su cui si tornerà più avanti.

\section{IL RUOLO DELLA SCIENZA GIURIDICA}

In questo complesso processo di ristrutturazione della res publica, di ridefinizione dei centri politici, quale fu il ruolo dei giuristi? Mantennero quella caratteristica di tecnicità e separatezza dal potere politico o cambiò qualcosa? Pur senza perdere quel carattere, qualcosa mutò e anche significativamente. Augusto aveva piena consapevolezza che quello romano era un 'diritto di giuristi' e non di legisti. Per questo aprì una fase nuova segnata da una loro chiamata alla partecipazione al potere. Ci provò persino con un dissidente del calibro di Labeone, uno dei maggiori giuristi del tempo, che però rifiutò senza andare incontro ad alcuna conseguenza negativa, ma in generale ci fu la disponibilità a stare a diverso titolo nell'orbita imperiale.

Assunsero cariche pubbliche, accettarono lo ius respondendi ex auctoritate principis, cioè la facoltà di emanare responsi rafforzata dall'auctoritas di Augusto, riannodando così quel filo spezzatosi tra prudentes e populus da cui scaturiva il riconoscimento della loro funzione sociale e creatrice di diritto, quell'auctoritas di cui parlava Cicerone. Ma soprattutto parteciparono come consiglieri giuridici all'esercizio diretto del potere nel consilium principis, introiettando dentro quel sistema di potere la loro scienza e le loro tecniche. Non passerà molto tempo infatti perché, oltre alla sostanza, il metodo casistico dei prudentes romani divenisse anche il metodo di intervento normativo del principe, soprattutto al giungere di quella, giustamente considerata, la fase più alta e luminosa della scienza giuridica romana nell'età dei Severi, in cui i migliori giuristi, Papiniano, Paolo, Ulpiano, Modestino, assunsero un notevole peso politico, trovandosi ai massimi vertici della burocrazia imperiale.

La scienza giuridica fu, dunque, fondamentale, e Augusto investì molto nell'attrazione verso il regime. Ebbe dalla sua parte Ateio Capitone, il costituzionalista del tempo, che condivise il progetto augusteo di rifondazione della res publica ed esercitò un ruolo fondamentale nella sistemazione del potere pubblico e, come dicevamo prima, nella teorica della sovranità popolare e della lex, come fa fede la sua celebre definitio:

Gell. N.A. 10, 20, 2: Ateius Capito, publici privatique iuris peritissimus, quid 'lex' esset, hisce verbit definivit: "Lex" - inquit - "est generale iussum populi aut plebis rogante magistratu”. 
Non è privo di significato che Gellio ricordasse Ateio Capitone ${ }^{20}$, peritissimus publici privatique iuris, un autorevole giurista capace di dominare i due settori del diritto, non solo quello tradizionale privatistico, ma pure lo ius publicum, quale sapere specialistico relativo allo status rei Romanae (D. 1, 1, 1, 2 [Ulp. 1 inst.]), che andò via via acquistando un peso rilevante, man mano che l'apparato istituzionale e amministrativo cresceva, accompagnato anche da una maggiore articolazione del potere nella sua dislocazione territoriale.

Verrebbe da chiedersi oggi dove siano i giuristi, quale sia il loro ruolo, a cosa sia dovuta la loro grave assenza soprattutto nei luoghi della rappresentanza politica, nelle assemblee titolari della produzione legislativa, perché si accetti ancora la compressione mortificante tra il potere politico sovente inadeguato e quello dei tribunali.

\section{LA VOCAZIONE UNIVERSALISTICA: UN IMPERO MULTIETNICO, MULTILINGUE, MULTICULTURALE, DAL PLURALISMO RELIGIOSO E NORMATIVO}

Ma torniamo ad Augusto. Il principe e la classe dirigente chiamata e formata accanto a sé sapevano di dover governare un pluriversum, in cui la res publica mostrava una sostanza complessa e composita quasi come una costellazione di città o un reticolo di entità statuali di diverso livello e di sovranità diseguale e subalterne a quella romana: civitates di differente statuto giuridico, liberae ac immunes, foederatae, regna amici, ecc. L'obiettivo gigantesco era quello di plasmare un governo mondiale, o se mi è permesso una 'global governance'. L'uso di simili espressioni, naturalmente, lungi dall'essere un vezzo, risponde a una banale esigenza di comodità rispetto a un orizzonte di mondializzazione indotto dal concetto antico di confine molto diverso da quello moderno, o forse meglio dall'assenza dello stesso. Secondo la concezione romana, infatti, l'impero coincideva con il mondo o viceversa ${ }^{21}$, oltre la demarcazione romana c'era l'altro, l'alterità, ma si era fuori dalla civiltà, cioè il barbaricum. Pertanto, rispetto all'essenza di un orbis consistente in un universo complesso, diversamente integrato e plurale di popoli, etnie, lingue, religioni, culture e tradizioni e ordinamenti giuridici diversi, la funzione esplicata dalle frontiere, da non intendere in senso esclusivamente militare ${ }^{22}$, era fondamentale. Non a caso, e da tempo, la migliore letteratura storiografica in tema di frontiere e confini ha dimostrato come questi debbano piuttosto essere intesi come significative aree di interazioni sociali e culturali ${ }^{23}$.

${ }^{20} \mathrm{Mi}$ limito a segnalare i contributi di S. RoNCATI, Caio Ateio Capitone e i Coniectanea (Studi su Capitone, I), in SDHI 71 (2005) 271 ss.; E. STOLFI, 'Plurima innovare instituit'. Spunti esegetici intorno al confronto tra Labeone e Capitone in D. 1.2.2.47 (Pomp lib. Sing. ench.), in Studi per G. Nicosia, VIII, Milano 2007, 57 ss.; P. BuOngIORNO, Ateii Capitones, in Iura 59 (2011) 195 ss.; ID., C. Ateius Capito. Appunti per una biografia politica, in Scritti per A. Corbino (a cura di I. Piro), I, Tricase (LE) 2016, 413 ss.; O. LiCANDRO, Augusto e la res publica imperiale, cit., passim, praecipue 291 ss.

${ }^{21}$ Da ultimo S. RoDA, Imperium sine fine e i confini dell'Impero tra ideologia, propaganda e Realpolitik, in AA.VV., Roma e $i$ 'diversi'. Confini geografici, barriere culturali, distinzioni di genere nelle fonti letterarie ed epigrafiche fra età repubblicana e Tarda Antichità, a cura di C. Giuffrida, M. Cassia e G. Arena, Milano 2018, 73 ss.

${ }^{22}$ Aspetto invece prediletto da A.S. LEWIN, Popoli, terre, frontiere dell'Impero romano. Il vicino Oriente nella tarda antichità. I: Il problema militare, Catania 2008, 109 ss.

${ }^{23}$ F.J. TURNER, La frontiera nella storia americana, Bologna 1975, 31 ss.; O. LATTIMORE, La frontiera. Popoli ed imperialismi alla frontiera tra Cina e Russia, Torino 1970, 49 ss.; J.B. WARD-PERKINS, Frontiere politiche e frontiere culturali, in AA.VV., La Persia e il mondo romano, Accademia dei Lincei, Quaderno 76, Roma 1966, 395 ss.; M. MAZZA, Identità etniche e culture locali sulla frontiera dell'Eufrate (II-IV sec. d.C.). Uno studio sui contatti culturali, in ID., Cultura guerra e diplomazia nella Tarda Antichità. Tre studi, Catania 2005, 13 ss. 
$\grave{E}$ in questa fase, e in relazione a determinate questioni di particolare delicatezza, che possiamo misurare il peso dei prudentes romani, e a tal proposito prendo in prestito alcune efficaci considerazioni, esordio di un agile e illuminante saggio, di Natalino Irti: «Soggetti, cose, atti abitano nello spazio. Ognuno di essi è individuato da un luogo e riceve un predicato di posizione. Il linguaggio giuridico è tutto intriso di richiami spaziali: dimora, residenza, domicilio delle persone fisiche; sede delle persone giuridiche; confini di terre e di altri beni immobili; contiguità o vicinanza di fondi; luoghi di conclusione di accordi, di adempimenti di doveri, di esercizio di diritti. L'applicazione di una o altra norma appare congiunta ai luoghi dell'aria e del mare, sui quali la superficie terrestre quasi si espande e irradia. C'è, nel profondo nascere e svolgersi del diritto, un legame terrestre, un'originaria necessità dei luoghi» ${ }^{24}$. Irti ci consegna una elementare considerazione: qualunque sia l'ordinamento giuridico preso in considerazione, per spazio fisico e per tempo, il diritto ha bisogno di spazi e luoghi.

Se vale ancora oggi, doveva ancor più valere allora. Davanti alla necessità di amministrare uno spazio di dimensioni gigantesche, Augusto e la nuova classe dirigente si trovarono dinanzi a questa nuova e prorompente esigenza. Del resto, Augusto stesso assolve a tale compito di ricognizione dello spazio e di riorganizzazione, quando nelle sue Res Gestae annota la deduzione di 28 colonie in Italia, e altre in Africa, Asia, ecc., la riconquista e riconsegna di numerosi territori all'imperium populi Romani. Il princeps in persona ${ }^{25}$ e la nuova classe dirigente, dunque, furono costrette a inventare mezzi sempre più avanzati di censimento della popolazione, di misurazione delle distanze, di rilevazione topografica e catastale, si crearono circoscrizioni amministrative con i loro strumenti (i 'Regionari'), si elaborarono criteri di relazioni della persona con luoghi e territori. Insomma, un immenso lavoro collettivo, un 'inventario del mondo', per utilizzare il titolo di un bel libro di Claude $\mathrm{Nicolet}^{26}$, che pose le basi per una nuova geografia amministrativa al servizio del governo del mondo.

\section{PERSONA E TERRITORIO: IL DOMICILIUM}

Proprio in questo arco cronologico i giuristi, impegnati nella costruzione di un nuovo impero mondiale, riflettono su grandi temi, come la cittadinanza, escogitano nuovi strumenti di governo e di criteri di relazione tra persona e territorio nell'ultimo secolo repubblicano. Vi è l'intera scienza giuridica in campo per decifrare la nuova realtà, per tradurre in principi e regulae coerenti, e contribuire così alla costruzione della disciplina giuridica dello spazio della nuova forma rei publicae.

I testi dei giuristi romani sopravvissuti grazie a quello straordinario giacimento che sono i Digesta di Giustiniano sono generosi ed eloquenti, a cominciare dal domicilium. È proprio negli ultimi cinquant'anni a.C. e nei primi decenni della genesi del principato che quel tema diventa oggetto di dibattito. E mentre la scuola serviana, con Alfeno Varo, cesellava una definizione di domicilium:

D. 50, 16, 203 (Alf. Var. 7 dig.): In lege censoria portus Siciliae ita scriptum erat: «servos quos domum quis ducet suo usu, pro is portorium ne dato». Quaerebatur, si quis a Sicilia servos Romam mitteret fundi instruendi causa, utrum pro his hominibus portorium dare deberet nec ne. Respondit duas esse in hac scriptura quaestiones, primam quid esset «domum ducere», alteram, quid esset «suo usu ducere». Igitur

\footnotetext{
${ }^{24}$ N. IRTI, Norma e luoghi. Problemi di geo-diritto, Roma-Bari 2002, 3.

${ }_{25}$ Divisio orbis terrarum 15: orbis dividitur tribus nominibus: Europa, Asia, Libya vel Africa. Quem Divus Augustus primus omnium per chorographiam ostendit.

${ }^{26}$ C. NiCOLET, L'inventario del mondo. Geografia e politica alle origini dell 'impero romano, Roma-Bari 1989.
} 
quaeri soleret, utrum, ubi quisque habitaret sive in provincia sive in Italia, an dumtaxat in sua cuiusque patria domus esse recte dicetur. Sed de ea re constitutum esse eam domum unicuique nostrum debere existimari, ubi quisque sedes et tabulas haberet suarumque rerum constitutionem fecisset,

con una visione che ritroviamo riflessa nella nostra contemporanea definizione civilistica dell'istituto (art. 43 c.c.: Il domicilio di una persona è nel luogo in cui essa ha stabilito la sede principale dei suoi affari e interessi), quale luogo ove si realizza la vita della persona e si concentrano i propri interessi, altri si interrogavano sulla legittimità del domicilio doppio o plurimo osservando la mutata realtà sociale ed economica imperiale: traffici commerciali intensi, presenza radicata di cittadini in ogni angolo del bacino del Mediterraneo a svolgere attività economiche, spingevano i giuristi romani a dibattere.

E si dividevano: se una maggioranza era favorevole, all'opposizione, invece, si schierava apertamente Labeone, espressione del conservatorismo repubblicano e giurisprudenziale. E si affinavano principi e regulae, sino a giungere ad affiancare al mero criterio materiale dell' $h a b i$ tatio o della strutturazione della propria attività commerciale, l'indagine della volontà, l'animus, della persona su quale luogo considerasse davvero il proprio domicilio ${ }^{27}$.

Al tempo stesso, il domicilium, oltre che nella sua essenza di luogo di imputazione degli affari e delle relazioni giuridiche, veniva preso in considerazione da un'altra e più delicata angolazione, cioè quale sfera personale riservata alla persona e come tale da proteggere da illegittime intrusioni materiali, persino violente. Basta una semplice analisi lessicale per comprendere la ricchezza della riflessione dei giuristi romani e della straordinaria eredità lasciataci. Emblematico, un testo di Ulpiano, relativo all'interpretazione della lex Cornelia de iniuriis che puniva in sede criminale le violazioni di domicilio aggravate dall'uso violenza ${ }^{28}$. Poiché nel testo della legge si usava domus piuttosto che domicilium, Ulpiano, che richiamava i termini di un dibattito di cui fu protagonista ancora una volta Labeone, precisava che l'interpretazione di domus dovesse essere estensiva e non restrittiva, non limitata alla domus di proprietà, ma a qualunque abitazione in cui si trovasse, a qualsivoglia titolo giuridico, l'individuo vittima della violazione violenta del domicilio.

Un'analisi del lessico giuridico romano in materia porterebbe a risultati interessanti: in alcuni casi si usa il termine domus, altre volte domicilium, altre volte ancora ci si riferisce all' $h a$ bitatio, cioè al mero fatto materiale di abitare in un certo luogo, e così via; in altri termini, si assiste a un continuo slittamento semantico che sembrerebbe deporre per uno stato di elaborazione della materia ancora grezzo, e una sostanza giuridica ancora confusa.

Ma se conducessimo una medesima analisi semantica sulla disciplina giuridica contemporanea avremmo una sorpresa. Partiamo dall'ordinamento giuridico italiano con l'art. 43 del codice civile italiano del 1942:

Il domicilio di una persona è nel luogo in cui essa ha stabilito la sede principale dei suoi affari e interessi. La residenza è nel luogo in cui la persona ha la dimora abituale.

L'influenza della concezione del domicilio romano è evidente: la sede principale degli affari e degli interessi (ricordiamo la definitio alfeniana di domus quale sede in cui sono conservate le scritture contabili, quindi un chiaro riferimento agli affari e alla loro dimensione giuridica) deve essere tenuta distinta dalla dimora abituale. Ma, passando da una dimensione privatistica a una pubblicistica, il quadro muta ed è agevole osservare come nel nostro ordinamento residui un approccio unitario tra residenza e domicilio, che sembra tendere a non distinguere tecnicamente

${ }^{27}$ D. 50.1 .5 ; D. 50.1.6.2; D. 50.1.27.2.

${ }^{28}$ D. $47.10 .5 p r .-5$. 
le due figure ai fini della tutela della sfera della persona. Ciò appare evidente nelle formulazioni dell'art. 14 della Costituzione:

\begin{abstract}
Il domicilio è inviolabile. Non vi si possono eseguire ispezioni o perquisizioni o sequestri se non nei casi e modi stabiliti dalla legge secondo le garanzie prescritte per la tutela della libertà personale. Gli accertamenti e le ispezioni per motivi di sanità e di incolumità pubblica o a fini economici e fiscali sono regolati da leggi speciali;
\end{abstract}

nella sua connessione con il dispositivo dell'art. 614 del codice penale:

\begin{abstract}
Chiunque s'introduce nell 'abitazione altrui, o in un altro luogo di privata dimora, o nelle appartenenze di essi, contro la volontà espressa o tacita di chi ha il diritto di escluderlo, ovvero vi s'introduce clandestinamente o con l'inganno, è punito con la reclusione fino a tre anni. Alla stessa pena soggiace chi si trattiene nei detti luoghi contro l'espressa volontà di chi ha il diritto di escluderlo, ovvero vi si trattiene clandestinamente o con inganno. Il delitto è punibile a querela della persona offesa. La pena è da uno a cinque anni, e si procede d'ufficio, se il fatto è commesso con violenza sulle cose, o alle persone, ovvero se il colpevole è palesemente armato.
\end{abstract}

La prima delle due formulazioni si riferisce più direttamente al domicilio, intendendo il luogo in cui una persona ha stabilito la sede principale dei suoi affari e interessi, secondo l'art. 43 del codice civile italiano; la seconda, invece, più in generale contempla qualunque luogo riconducibile a una persona (abitazione altrui o privata dimora), la cui violazione ingiustificata configurerebbe un'intrusione illecita nella sfera individuale.

E, infine, a completamento della sinossi può aggiungersi anche l'art. 12 della Dichiarazione universale dei diritti dell'uomo:

\footnotetext{
Nessun individuo può essere sottoposto a interferenze arbitrarie nella sua vita privata, nella sua famiglia, nella sua casa, nella sua corrispondenza, né a lesioni del suo onore e della sua reputazione. Ogni individuo ha diritto a essere tutelato dalla legge contro tali interferenze o lesioni.
}

Mentre con la rivoluzione tecnologica e con il poderoso impatto sulla vita sociale degli strumenti informatici e del $w e b$, ci si è proiettati su dimensione diversa in cui tutto è più astratto, meno materiale, lo spazio diventa indefinibile, come si legge nella formulazione di una nuova fattispecie di 'violazione del domicilio', contenuta dall'art. 615 ter del codice penale italiano:

Chiunque si introduce in un sistema informatico o telematico protetto da misure di sicurezza ovvero vi si mantiene contro la volontà espressa o tacita di chi ha diritto di escluderlo è punito con la reclusione fino a tre anni.

Introdursi clandestinamente in un server, in un computer, in una cloud, o in cos'altro, per quanto lontanamente assimilabile, configura oggi una violazione di domicilio. Chiunque può accorgersi che tutte queste disposizioni differenti e diversamente efficaci sono accomunate da un singolare slittamento semantico: casa, sede principale, abitazione, luogo di privata dimora, dimora abituale; mentre lascio fuori quelle informatiche o telematiche. Su queste basi, allora, può davvero parlarsi di incertezza? Erano allora soltanto i giuristi romani tardorepubblicani ad apparire incerti o confusi? La risposta è no. Al contrario, a proposito degli strumenti di inquadramento e di relazione tra persone e territorio, è noto che gli interessi in gioco producono effetti prismatici assorbenti e di assoluto interesse, e tali furono quelli che si determinarono man mano che si procedeva alla costruzione e razionalizzazione dello 'Stato imperiale' romano. 


\section{IL CRITERIO DELL'URBANITAS}

Il criterio su cui si fondava il rapporto tra una persona e una comunità cittadina consisteva nell'urbanitas, termine evocante, da un canto, un essenziale tratto identitario culturale della cittadinanza romana e, da un altro, la relazione tra una persona e un territorio. Ma in cosa consisteva l'urbanitas? Sull'affinamento dell'urbanitas la scienza giuridica fu decisiva. In particolare, può richiamarsi un passo assai significativo di Ulpiano, che contribuisce a chiarire appunto cosa entrasse in gioco nella valutazione della relazione tra una persona e il territorio, o i territori, su cui insisteva:

D. 50, 1, 27, 1 (Ulp. 2 ad ed.): Si quis negotia sua non in colonia, sed in municipio semper agit, in illo vendit, emit contrahit, in eo foro balineo spectaculis utitur, ibi festos dies celebrat, omnibus denique municipii commodis, nullis coloniarum fruitur, ibi magis habere domicilium, quam ubi colendi causa deversatur.

Ulpiano prendeva in considerazione il caso di chi possedeva un rapporto ambiguo con due strutture urbane, una colonia o un municipio: in una città conduceva i propri affari negoziali, frequentava il foro e le terme, partecipava agli spettacoli e alle feste, in un'altra invece concretizzava semplicemente una materiale habitatio. Il giurista severiano, com'è evidente, ai fini dell'attribuzione del domicilium individuava un preciso indicatore della qualità del legame di un individuo con il territorio, e cioè la partecipazione alla vita economica, sociale e culturale di una città.

Il tema ricorre in un altro testo di un altro giurista severiano, Modestino:

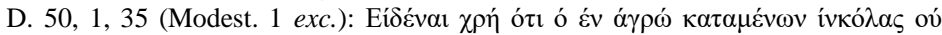
vo ívкó $\lambda \alpha \varsigma$

Il caso affrontato è quello della determinazione del domicilio di chi, abitando in un fondo rustico, non si avvaleva delle prerogative, dei vantaggi, dei servizi, insomma dei commoda, della città. Ora, è utile ricordare le numerose disposizioni ricorrenti negli statuti municipali in cui si contemplavano ed enumeravano proprio tali aspetti: basta scorrere, per esempio, la lex Irnitana in cui si menzionano cenae e ludi (cap. 77); distribuzioni di denaro (cap. 79); spectacula (cap. 81); epula, viscerationes (cap. 92 ${ }^{29}$. Però, non è che a questa soluzione si fosse giunti soltanto nel III secolo d.C.: in realtà, le posizioni di Ulpiano e Modestino confermano un preciso e univoco orientamento di continuità dei prudentes romani attenti non tanto, o almeno non soltanto, al luogo dove banalmente si soggiornasse, cioè dove diremmo volgarmente oggi si andasse a dormire, ma alla sostanza reale e qualificata del rapporto tra individuo e comunità ${ }^{30}$.

Si trattava, insomma, di uno dei cardini su cui ruotava l'essenza della romanità, cioè l'urbanitas quale stile di vita urbano che soltanto una struttura cittadina era in grado di offrire ai propri membri: «un tessuto urbanistico fatto di edifici privati e pubblici con strutture in grado di assicurare la vita associata nel profilo economico, culturale, politico». L'attenzione anche alle

\footnotetext{
${ }^{29} \mathrm{Su}$ questi capita rinvio per tutti a F. LAMBERTI, «Tabulae Irnitanae». «Municipalità»e «Ius Romanorum», Napoli 1993, passim.

${ }^{30} \mathrm{Su}$ questi testi vedi principalmente O. LICANDRO, Domicilium e incolae tra repubblica e principato, in AA.VV., Étrangers dans la cité romaine. " "Habiter une patrie": des incolae de la République aux peuples fédérés du BasEmpire». Actes du Colloque de Valenciennes (14-15 octobre 2005), Rennes 2007, 66 ss.; ID., Pomponio e L'incola. Osservazioni su D. 50.16.239.2 (Pomp. l. sing. ench.) alla luce di Lex Urs. cap. 98 e Lex Irn. cap. 83, in fil...a. Scritti per Gennaro Franciosi, a cura di F.M. D’Ippolito, II, Napoli 2007, 1381 ss.; e L. GAGLIARDI, Osservazioni in tema di domicilio degli incolae. La distinzione tra incolae di città e incolae di campagna, in AA.VV., Gli Statuti Municipali (a cura di L. Capogrossi Colognesi, E. Gabba), Pavia 2006, 647 ss.
} 
strutture materiali, ove si svolgeva la vita cittadina, costituì sempre una delle fondamenta ideologiche della città e della cittadinanza: templi, fori, teatri, terme, fontane, ginnasi, scuole, definivano un modello non solo e non tanto urbanistico quanto assai più profondamente culturale ${ }^{31}$. Lo schema del modello era già offerto da Cicerone che nel De officiis, in un tentativo di inquadramento definitorio del civis, teneva strettamente congiunti tre aspetti (Cic. de off. 1, 17, 53: multa enim sunt civibus inter se communia, forum, fana, porticus, viae, leges, iura, iudicia, suffragia, consuetudines praeterea et familiaritates cum multis res rationesque contractae).

Dunque, dagli elementi istituzionali, quali il diritto e i giudici e la politica (leges, iura, iudicia, suffragia), ai rapporti interpersonali e di affari ('consuetudines praeterea et familiaritates cum multis res rationesque contractae'), alle strutture materiali cittadine (forum, fana, porticus, viae), nonostante la distanza di diversi secoli, da Cicerone a Ulpiano, la linea non appare affatto mutata. Nelle righe ciceroniane troviamo la più incisiva dimostrazione che la città non è un semplice fatto materiale, non è solo pietre, ma anche, direi soprattutto, modelli culturali, moduli politico-organizzativi, idee; ma niente di tutto ciò potrebbe allignare al di fuori di uno spazio urbano organizzato secondo il tempo della politica, dello ius, degli affari. Sempre quei medesimi tre aspetti cesellarono a tutto tondo lo status di un cittadino e il suo rapporto con una comunità urbana, e il cuore del problema restava appunto quello della cultura urbana, misura di una compiuta romanizzazione, di norma requisito per il conseguimento della cittadinanza.

\section{CitTtadinanza: Città e Campagne}

La città era l'essenza dell'impero romano, la sua stessa base senza la quale non sarebbe stato nulla e la campagna non era altro che una parte del suo territorio. Nel suo incompiuto Die Stadt, Max Weber ha avvertito come nella definizione di città la grandezza non fosse carattere decisivo $^{32}$. A prescindere dalle dimensioni, ovunque arrivasse, l'impero creava città o nei territori privi di ogni cultura urbana organizzava centri e comunità di villaggio, diversamente denominati (fora, vici, loci, conciliabula, oppida, castella) secondo una tipologia variabile in base ad alcuni tratti fisionomici prevalenti, ma il cui approdo allo stadio finale della forma della città era l'esito solo eventuale di un processo graduale di crescita e trasformazione ${ }^{33}$.

Il paradigma della città con il suo modello culturale sintetizzato dal termine urbanitas, lo ritroviamo in un Elogio di Roma pronunciato da un giovane e brillante retore intorno alla metà del II secolo d.C., Elio Aristide ${ }^{34}$. Nella visione aristidea l'urbanitas si condensava nelle peculiarità di Roma, appunto città per eccellenza, munita di quella particolare fisionomia grazie alla quale le si assegnava il primato nel confronto con le poleis e gli imperi del passato; un tratto che recava in sé la ricchezza dei consumi e delle abitudini, la bontà delle istituzioni e del governo.

${ }^{31}$ Cfr. Tac. Agr. 21.

${ }^{32}$ M. WEBER, Economia e società. L'economia in rapporto agli ordinamenti e alle forze sociali. La città (a cura di W. Nippel), Roma 2003, 3 s., osservava come nella Russia dei suoi tempi ci fossero villaggi con diverse migliaia di abitanti di gran lunga più grandi di alcune città antiche, abitate soltanto da qualche centinaio di individui.

${ }^{33}$ V. GIUFFRÈ, La struttura politico-costituzionale e il territorio, in AA.VV., Città territorio e diritto privato nei primi due secoli dell'impero. Atti del convegno internazionale di diritto romano, Copanello 5-8 giugno 2002 (a cura di F. Milazzo), Soveria Mannelli 2010, 26, ha giustamente sottolineato lo scarso valore tecnico sotto il profilo istituzionale di questa tipologia di insediamenti, da un lato, refrattari «alla latinizzazione/romanizzazione amministrativa dell'area» e, dall'altro, ritenuti da Roma inadeguati «a sostenere gli oneri dell'autogoverno, e inaffidabili nell'esazione tributaria».

${ }^{34}$ Sul retore si rinvia a ELIO ARISTIDE, A Roma (traduzione e commento a cura di F. Fontanella, introduzione di P. Desideri), Pisa 2007; e i saggi del volume collettaneo AA.VV., Elio Aristide e la legittimazione greca dell'impero di Roma, a cura di P. Desideri e F. Fontanella, Bologna 2013. 
Elio Aristide tratteggia un quadro assai definito nel suo perimetro retorico, ove i confini dell'impero erano i confini del mondo, la pace e il paradigma della città costituivano l'architrave della sua rappresentazione di un impero-mondo, di un'urbs fattasi orbis, communis patria, come aveva già scritto Cicerone: «Il mondo intero è stato trasformato in un delizioso giardino», le campagne sono sicure «le fumate che si levavano dalle pianure, i fuochi di segnalazione per amici e nemici sono svaniti», i mari adesso sono solcati «da navi mercantili invece che da triremi da guerra», l'impero è segnato da una moltitudine di città «le coste del mare e le regioni dell'interno ne sono gremite», e dovunque «ginnasi, fontane, templi, manifatture, scuole». Alla stessa stregua di Cicerone, Elio Aristide individuava il merito principale dell'impero romano nell'aver fatto della città, della polis, il paradigma dell'assetto politico e istituzionale e della stessa civiltà.

In tal modo il processo di uniformazione trovava un suo cardine fondamentale e si compiva nella globalità dell'impero: anche per i territori orientali la polis diveniva la forma organizzativa della convivenza civile e dello sviluppo economico; e su queste basi, non a caso, si radicava il consenso delle élites di cultura greca. È utile rileggerne l'affresco nella pagina aristidea: «[...] Il mare come una cintura segna il centro dell'ecumene e allo stesso tempo del vostro impero; [11] e intorno al mare si stendono i continenti, grandi in grande spazio, che sempre vi forniscono in abbondanza qualcuno dei beni che da essi provengono. Qui confluisce da ogni terra e da ogni mare quello che generano le stagioni e producono le varie regioni, i fiumi, $i$ laghi, e le arti dei Greci e dei barbari; se uno vuole osservare tutte queste cose, bisogna o che se le vada a vedere viaggiando per tutta l'ecumene, o che venga in questa città. Infatti quando nasce e si produce presso ciascun popolo, non è possibile che non si trovi sempre qui addirittura in abbondanza. Tante sono le navi da carico che giungono qui trasportando tutti i prodotti da tutti i luoghi, in ogni stagione, in ogni volgere d'autunno, che l'Urbe sembra il laboratorio generale della terra. [12] E si possono vedere cosi tanti carichi dall'India e volendo anche dall'Arabia Felice, da potersi presumere che ormai a quei popoli gli alberi sia rimasti spogli, $e$ che anche loro debbano venire qui a cercare i loro stessi prodotti, nel caso che abbiano bisogno di qualcosa; inoltre tessuti babilonesi e ornamenti delle regioni barbare più lontane arrivano in molto maggiori quantità, e molto più facilmente, che se si dovesse venire ad Atene portando qualche prodotto di Nasso o di Citno; e l'Egitto, la Sicilia e la parte fertile dell'Africa sono come vostri poderi. [13] Gli arrivi e le partenze delle navi si susseguono senza posa, così che c'è da meravigliarsi non tanto che il porto, quanto che il mare stesso riesca, se pure riesce, a contenere un così gran numero di imbarcazioni. E veramente si può dire, come diceva Esiodo degli estremi confini dell'Oceano - che c'è un luogo dove tutto confluisce in un unico principio e in un 'unica fine - che qui tutto converge, commerci, navigazioni, agricoltura, metalli lavorati, tutte quante le arti che ci sono o che ci sono state, tutto quanto è prodotto o generato dalla terra. Quello che non si riesce a vedere qui, non rientra nell'ordine delle cose che sono esistite o esistono; per questo non è facile decidere se sia più l'Urbe a superare le città a lei contemporanee, o il suo impero a superare tutti gli imperi del passato» (Ael. Arist. Elogio di Roma 10-13).

Le élites cittadine trovavano voce in Elio Aristide per descrivere se stesse come la vera 'spina dorsale' dell'impero romano: «di qui l'ambivalenza di un elogio di città che è l'elogio della stessa costruzione imperiale nella sua dimensione territoriale complessiva, come spazio politico, amministrativo, militare e anche economico unitario ${ }^{35} \mathrm{e}$, certamente, in quanto dilatazione del paradigma della città. Roma era l'impero, non tanto la capitale, e le casate imperiali e le classi dirigenti centrali furono sempre consapevoli del fatto che le mille città dell'impero fossero il fulcro politico del consenso ed economico delle tante reti regionali di cui si componeva il panorama mercantile.

${ }^{35}$ E. LO CASCIO, Roma come «mercato comune del genere umano», in AA.VV., Elio Aristide e la legittimazione greca dell'impero di Roma, a cura di P. Desideri e F. Fontanella, Bologna 2013, 193. 
Corre, infatti, un unico filo rosso nei secoli imperiali, che per comodità facciamo andare da Cicerone in avanti. Quando nel 177 d.C. Marco Aurelio decise di concedere la cittadinanza romana a esponenti di una tribù nomade berbera (gli Zegrenses), nella motivazione del provvedimento, fondata sulla sua comprovata e assoluta fedeltà all'impero, ne sottolineava l'eccezionalità, perché Roma era restia a darla a persone appartenenti a organizzazioni tribali seminomadi, come nel caso in questione, lontane dallo standard di romanizzazione espresso, in particolare, dal modello di vita associata in città ${ }^{36}$.

Città e urbanitas furono sempre connessi al tema, perennemente infuocato, della cittadinanza (e, perciò, della romanizzazione). Dinanzi alla tumultuosa ansia palingenetica e di pacificazione, Augusto intuì abilmente come uno snodo centrale passasse dalla necessità di invertire una tendenza in tema di cittadinanza. La linea politica di geloso esclusivismo in materia di concessione di cittadinanza, praticata con rigore per secoli dai gruppi dirigenti repubblicani, era ormai davvero insostenibile. Del resto, aveva prodotto disastri incalcolabili, come il bellum sociale nell'ultimo secolo della repubblica, che si chiuse con la vittoria militare romana ma con la vittoria politica degli alleati ammessi allo ius civitatis $^{37}$.

In un celeberrimo dialogo, riportato da Cassio Dione, di Augusto con i suoi uomini più fidati Agrippa e Mecenate (il militare e il politico intellettuale), quest'ultimo sollecitava il princeps a chiamare alla partecipazione al potere il notabilato locale: «dichiaro che tutti devono essere chiamati a prendere parte al governo, in modo tale che, partecipando alla pari anche in questo, siano nostri fedeli alleati come se vivessero in un'unica città, che definiamo nostra, considerandola tutt'uno con i loro campi e i loro villaggi» (Cass. Dio 52, 19, 6). In questa idea antica, non importa poi se davvero ascrivibile a Mecenate o ad Augusto o allo stesso Cassio Dione, vi è un nucleo di assoluta modernità, quello del 'villaggio globale': relazioni tra gruppi dirigenti di comunità lontane dislocate anche agli estremi di un impero sterminato, come se stessero in un'unica città. Una visione moderna che mirava a tenere insieme élites e popolo, città e campagne, cittadini urbanizzati e masse rurali.

Augusto apriva, così, il varco alla doppia cittadinanza per includere le élites locali in una visione comune e di partecipazione al potere. Per quanto la situazione sia molto diversa, è indubbia l'analogia con uno dei temi contemporanei più scottanti sul campo europeo, appunto la frattura tra centro e periferia, tra élites e popolo. Nell'attuale temperie mondiale, l'irruzione potente dei nuovi nazionalismi declinati nella forma del 'sovranismo' hanno prodotto fatti politici enormi e, conseguentemente, sollevato un gigantesco dibattito. Sovviene, istintivamente, l'esito referendario britannico della Brexit che ci ha plasticamente rappresentato la divaricazione tra città e campagna nel mondo anglosassone. Abbiamo visto soccombere la visione, e la voglia, di cosmopolitismo, di integrazione, di unità pur nella diversità, dinanzi all'arrembante pulsione centrifuga, scatenata da propagande fondate su paure, egoismi, nazionalismi.

L'Italia non ne è rimasta immune. Gli esiti delle recenti vicende politiche hanno così stimolato un libro intelligente, acuto, The Game (pubblicato per i tipi di Einaudi, 2018), di Ales-

\footnotetext{
${ }^{36}$ Sulla Tabula Banasitana vedi per tutti E. VOLTERRA, La Tabula Banasitana. A proposito di una recente pubblicazione, in BIDR 77 (1974) 407 ss.; E. MIGLIARIO, Gentes foederatae. Per una riconsiderazione dei rapporti romanoberberi in Mauretania Tingitana, in RAL 10 (1999) 427 ss.; C. GIACHI, La Tabula Banasitana: cittadini e cittadinanza ai confini dell'Impero, in AA.VV., Civis/Civitas, Cittadinanza politico-istituzionale e identità socio-culturale da Roma alla prima età moderna. Atti del Seminario internazionale di Siena-Montepulciano, 10-13 luglio 2008 (a cura di C. Tristano, S. Allegria), Montepulciano (SI) 2009, 17 ss.

${ }^{37}$ G. LuRASCHI, Foedus Ius Latii Civitas. Aspetti costituzionali della romanizzazione in Transpadania, Padova 1979; S. BARBATI, Gli studi sulla cittadinanza romana prima e dopo le ricerche di Giorgio Luraschi, 175 ss. [= in RDR 12 (2012) (www.ledonline.it)], e bibliografia ivi citata.
} 
sandro Baricco, su alcune coordinate discusse poi per qualche settimana sulle colonne del quotidiano La Repubblica ${ }^{38}$. Provo a riassumerne la sostanza. Baricco sostiene che il caos attuale derivi, sostanzialmente, dalla rottura del patto tra élites e la gente, e cioè nel fatto che «la gente concede alle élites dei privilegi e perfino una sorta di sfumata impunità, e le élites si prendono la responsabilità di costruire e garantire un ambiente comune in cui sia meglio per tutti vivere [...] una comunità in cui le élites lavorano per un mondo migliore [...]». Se poi invece accade il contrario, se la crisi, mentre divora economie e travolge intere masse popolari, non colpisce le élites, comprendiamo i termini del problema e della sua capacità di innescare processi dagli esiti imprevedibili: frantumazione della classe media, smarrimento dei suoi valori, rifiuto, se non disprezzo, delle istituzioni, perdita di identità, insorgenza di culture violente, tendenze secessionistiche, pulsioni di l'abbattimento del sistema.

Con tutti i dovuti distinguo, sono innegabili i tratti di affinità con alcuni momenti cruciali dell'antichità e, per il tema che si sta trattando, dei problemi che Augusto si trovò ad affrontare. $\mathrm{E}$, allora, se con tutta la cautela necessaria analizziamo freddamente le questioni, affiora subito la cifra alta della strategia politica augustea.

Attraverso un sapiente processo di integrazione nel sistema di governo, chiamate a fianco del nuovo protettore della res publica, le élites locali avrebbero accresciuto il corpo civico romano, assicurato una capillare adesione alle politiche imperiali, accelerato la romanizzazione delle loro comunità attraverso la diffusione del modello urbano romano e dei relativi costumi e idee, garantito loro un avanzamento sul piano dei benefici giuridici e un maggior benessere generale. Avrebbero in fin dei conti fatto uscire persino dalla marginalità periferica la loro comunità. In più, questione nient'affatto trascurabile, avrebbero garantito ordine. Non c'è dubbio che l'obiettivo di trasformare la potenza in ordine abbia costituito la cifra più alta e qualificante dell'azione augustea, la pietra angolare su cui costruire un nuovo e complesso edificio istituzionale adeguato alla massa critica di un impero sterminato che richiedeva un nuovo ordine mondiale. Insomma, un obiettivo del tutto diverso da quell'allontanamento delle comunità locali e periferiche che si potrebbe a prima vista pensare, e finalizzato invece a una piena integrazione e partecipazione di élites e popolo, naturalmente secondo lo stato e i canoni del tempo. Augusto mise in pratica questa linea politica con alcuni provvedimenti, di cui è sopravvissuta fortunatamente la documentazione epigrafica, come il III Editto ai Cirenei ${ }^{39}$ e l'Editto di Rhosos, con i quali concedeva appunto la cittadinanza romana alle élites locali, consentendo al tempo stesso di restare integrati nelle loro comunità, e, così, permettendo loro di avvalersi di nuclei normativi distinti e di tribunali diversi ${ }^{40}$.

Certo, potrebbe obiettarsi che l'esperienza augustea guardasse ai notabili più importanti di tutte le province, secondo il consiglio di Mecenate. Sembrerebbe, insomma, una testimonianza di disprezzo e di lontananza rispetto alle periferie, attenta alle élites, quindi orientata in direzione contraria al problema, ma la contraddizione è soltanto apparente, o almeno non lo è per quei tempi e in quel contesto. In realtà, l'impianto ideologico, poi alla base delle trasformazioni politico-istituzionali e amministrative, seguiva l'efficace linea strategica, suggerita dall'abile e raffinato consigliere, di costruzione del consenso attraverso l'attrazione nell'orbita del principe della migliore aristocrazia locale, delle classi possidenti delle città di Occidente e Oriente, come

\footnotetext{
38 A. BARICCO, The Game, Torino 2018; The Game Unplugged, Torino 2019. Ma si veda anche TH. HYLLAND ERIKSEN, Fuori controllo. Un'antropologia del cambiamento accelerato, Torino 2017; F. FUKUYAMA, Identità. La ricerca della dignità e i nuovi populismi, Milano 2019; CH. GuILluY, La società non esiste. La fine della classe media occidentale, Roma 2019.

${ }^{39}$ G. PurPurA, Edicta Augusti ad Cyrenenses (6/4 a.C.), in AA.VV., Revisione ed integrazione dei Fontes Iuris Romani Anteiustiniani (FIRA). Studi preparatori. I. Leges (a cura di G. Purpura), Torino 2012, 433 ss., praecipue 444 ss.

40 A. Raggi, Seleuco di Rhosos. Cittadinanza e privilegi nell'Oriente greco in età tardo-repubblicana, Pisa 2006, 94 ss.; O. LICANDRO, Doppio domicilio e doppia cittadinanza, cit., 168 ss.
} 
volano di sviluppo e, in definitiva, di accelerazione del processo di romanizzazione. L'emersione, dopo il secolo tragico delle guerre civili, di nuovi ceti possidenti disponibili a 'scambiare' fedeltà con pace e benessere produsse la formazione di una sorta di omogeneo notabilato mediterraneo sicuramente il principale beneficiario della crescita romana ma anche in grado di permettere in qualche misura una distribuzione di ricchezza, con fenomeni di 'delocalizzazioni' di attività produttive dall'Italia alle province ${ }^{41}$, e persino con incontestabili effetti benefici pure sui ceti meno abbienti. Perché nello spazio urbano, serbatoio e vivaio di saperi, competenze amministrative e politiche, depositi di ricchezze e propulsori economici, non vi erano solo élites ma si addensavano larghi ceti medi che appunto vivevano, aderendovi ideologicamente, secondo quel modello organizzativo politico ed economico urbano.

$\mathrm{Fu}$, appunto, inscindibile e biunivoco il nesso tra consenso e integrazione: non si aveva consenso se non si realizzava integrazione. Tale nesso fu declinato attraverso il modello urbano ed emerse grazie a un lungo e non sempre lineare processo di maturazione politica, e per la verità non del tutto attribuibile ad Augusto, come ci consente di osservare la documentazione sopravvissuta: si veda, ad esempio, quanto scritto da Cicerone a Quinto a proposito del suo proconsolato d'Asia (Cic. ad Quint. fr. 1, 1, 27). Ma ben oltre Cicerone, è indubbio il merito dell'azione augustea di consolidare quel nesso, anzi di assumerlo come strumento per plasmare la nuova res publica nelle forme dello 'stato municipale', per usare una felice espressione di Emilio Gabba ${ }^{42}$, rimodulata con altrettanto efficacia in 'impero municipale' da Luigi Capogrossi Colognesi ${ }^{43}$, quale trama sempre più complessa di relazioni tra centro e periferie improntata a un sistema di municipia e coloniae, a cominciare dall'Italia come si evince già da $R G D A 21,3^{44}$. Nella biografia augustea di Svetonio vi è un'annotazione preziosa: Augusto, dopo aver riordinato Roma e la sua amministrazione, fondò 28 colonie prevedendo per i decuriones il privilegio di una sorta di 'voto a distanza', cioè la possibilità di partecipare alle elezioni dei magistrati, pur restando nella propria città e inviando le tabellae elettorali il giorno di convocazione dei comizi elettorali ${ }^{45}$. Non conosciamo del tutto questo meccanismo di voto, un'ipotesi convincente è quella proposta da Claude Nicolet ${ }^{46}$, ma ciò che interessa è che la città assurge sempre a protagonista nella visione augustea. Nella città, territorio della politica e motore dell'economia, «luogo preferenziale per lo svolgimento dei diritti e dei doveri della nuova vita associata ${ }^{47}$, si affinavano forme e

${ }^{41}$ In tal senso E. Lo CASCIO, Forme dell'economia imperiale, in AA.VV., Storia di Roma. 2. L'impero mediterraneo. II. I principi e il mondo (dir. A. Schiavone), Torino 1991, 358 ss. Si legga pure ID., La "New Institutional Economics" e l'economia imperiale romana, in AA.VV., Storia romana e storia moderna. Fasi in prospettiva (a cura di M. Pani), Bari 2005, 313 ss.; ID., The Early Roman Empire: the State and the Economy, in AA.VV., The Cambridge Economic History of the Greco-Roman World (ed. W. Scheidel, I. Morris, R. Saller), Cambridge 2007, 619 ss.

${ }^{42}$ E. GABBA, Dallo stato-città allo stato municipale, in AA.VV., Storia di Roma. 2. L'impero mediterraneo. I. La repubblica imperiale (dir. A. Schiavone), Torino 1990, 697 ss. Ma si vedano anche gli studi dedicati al tema, contenuti in due raccolte, di U. LAFFI, Studi di storia romana e di diritto, Roma 2001; ID., Colonie e municipi nello Stato romano, Roma 2007.

${ }^{43}$ L. CAPOGRossi COLOGNESI, La genesi dell 'impero municipale, in Roma e America. Diritto romano comune 18 (2004) 243 ss. [= in ID., Scritti scelti, II, Napoli 2010, 939 ss.].

44 E. GABBA, L'impero di Augusto, in AA.VV., Storia di Roma. 2. L'impero mediterraneo. II. I principi e il mondo (dir. A. Schiavone), Torino 1991, 26 s.; vedi anche ID., La rifondazione di Salapia, in Athenaeum 71 (1983) 514 ss.; ID., Le città italiche del I sec. a.C. e la politica, in RSI 98 (1986) 653 ss.; ID., Municipium Augustum Veiens, in Athenaeum 86 (1988) 203 s.; ID., I municipi e l'Italia augustea, in AA.VV., Continuità e trasformazioni fra repubblica e impero. Istituzioni, politica, società, Bari 1991, 69-82; poi in Italia romana, Como 1994, 133-143.

${ }^{45}$ Svet. Aug. 46, 1: Ad hunc modum urbe urbanisque rebus administratis Italiam duodetriginta coloniarum numero eductarum a se frequentavit operibusque ac vectigalibus publicis plurifariam instruxit, etiam iure ac dignatione urbi quodam modo pro parte aliqua adeaquavit excogitato genere suffragiorum, quae de magistratibus urbicis decuriones colonici in sua quisque colonia ferrent et sub die comitiorum obsignata Romam mitterent.

${ }^{46}$ C. NiCOLET, L'inventario del mondo, cit., 240 ss.

${ }^{47}$ E. GABBA, Dallo stato-città allo stato municipale, cit., 709. 
strumenti di inquadramento e di amministrazione: catastazioni, censimenti, collocazione dei cittadini nelle classi di censo, registri di cittadini e non, o di particolari categorie ${ }^{48}$.

Tutto ciò fu uno dei lasciti più importanti del princeps, raccolto più o meno costantemente dai suoi successori, di cui è significativo il celebre discorso di Claudio per la lectio senatus di alcuni notabili della Gallia Comata (Tac. ann. 11, 23-25; CIL XIII, $1668=$ ILS $212=$ FIRA I, $43)^{49}$. Nessuno dubita che uno dei tratti fondamentali e costanti della civilitas romana consistette proprio «nella fondazione e nell'incremento di centri cittadini ${ }^{50}$. Persino in quel tornante di profonda svolta della storia imperiale interpretata dalla dinastia severiana (in particolar modo da Settimio Severo), troviamo solida conferma della stretta continuità della linea politica con quella augustea, messa in atto in particolare nel riordinamento dei territori nordafricani ${ }^{51}$. Non mi riferisco tanto alla creazione della nuova provincia di Numidia, quanto all'accrescimento e all'arricchimento urbanistico delle città esistenti, la concessione di statuti municipali e coloniali e dello ius Italicum a nuovi centri, quali fattori principali di sviluppo economico e demografico ${ }^{52}$.

Se un'iscrizione di Thugga del 313 d.C. celebrava Costantino come restitutor urbium (AÉ 2003, n. 2014) $)^{53}$, altrettanto significativa è l'ideologia della città espressa in un'iscrizione del III secolo d.C. di Tymandus nell'autocelebrazione di un imperatore autore dell'accrescimento del numero e del prestigio delle città:

ILS 6090, 11. 8-15 (FIRA I, 92): Cum itaque ingenitum nobis | sit, ut per universum orbem nostrum civi|tatum honor ac numerus augeatur eos|que eximie cupere videamus, ut civitatis $\mid$ nomen honestatemque percipiant, isdem $\mid$ maxime pollicentibus, quod apud se decu|rionum sufficiens futura sit copia, cre|didimus advendum ${ }^{54}$.

${ }^{48}$ Interessante la notizia del commentarius civitate Romana donatorum contenuta nel diploma AÉ 1999, n. 1250, 11. 310: Descriptum et recognitum ex petitione rescripta diplomi id quod infra scriptum est: Descriptum et recognitum ex commentario civitate donatorum divi Augusti et Tiberii Caesaris Augusti et C. Caesaris et divi Claudi et Neronis et Galba et divorum Augustorum Vespasiani et Titi et Caesaris Domitiani et divorum Augustorum Nervae et Traiani Parthici et Traiani Hadriani Antonini Pii (su cui vedi R. FREI-STOLBA, H. LIEB, Un diplôme civil: le fragment de Carnuntum, in ZPE 143 [2003] 243 ss.; da ultimo V. MAROTTA, Doppia cittadinanza e pluralità degli ordinamenti cittadini. La Tabula Banasitana e le linee 7-9 del Papiro di Giessen 40 col. I, in AG 236 [2016] 471 s.). Cfr. ILMaroc 94, ll. 22. In generale, vedi O. BEHRENDS, Die Rechtsregeln der Militärdiplome und das die Soldaten des Prinzipats treffende Eheverbot, in AA.VV., Heer und Integrationspolitik (hrsg. W. Eck, H. Wolff), Köln-Wien 1986, 123 ss.

49 Altrettanto sterminata è la letteratura sulla Tavola di Lione, per cui mi limito ai seguenti scritti e da essi risalire ad ulteriore bibliografia: PH. FABIA, La Table Claudienne de Lyon, Lyon 1929; P. SAGE, La Table claudienne et le style de l'empereur Claude: essai de réhabilitation, in REL 58 (1980) 274 ss.; P. BuONGIORNO, Senatus consulta Claudianus temporibus facta. Una palingenesi delle deliberazioni senatorie dell'età di Claudio (41-54 d.C.), Napoli 2010, 261 ss.; R. TURCAN, Claude de Lyon: un précurseur, in BIDR 107 (2013) 21 ss.

50 Così S. MazZarino, L'Impero romano, II, Roma-Bari 1988, 754; vedi anche M. FeliCI, Profili storico-giuridici del pluralismo cittadino in Roma antica, Roma 2013, 51 ss. Per altri aspetti si legga F.P. CASAvola, Il concetto di "Urbs Roma"; giuristi e imperatori romani, in Labeo 38 (1992) 20 ss. [= in ID., Sententia legum tra mondo antico e moderno. I. Diritto romano, Napoli 2000, 355 ss.].

${ }^{51}$ In particolare vedi O.D. CoRdovANA, Segni e immagini del potere tra antico e tardoantico. I Severi e la provincia Africa proconsularis, Catania 2007; G. PoMA, Nota su CIL, VIII, 9228: populis novis ex Africa inlatis, in AA.VV., Roma e $i$ 'diversi'. Confini geografici, barriere culturali, distinzioni di genere nelle fonti letterarie ed epigrafiche fra età repubblicana e Tarda Antichità, a cura di C. Giuffrida, M. Cassia e G. Arena, Milano 2018, 26 ss.

52 Troppo ideologicamente schematica è la visione di M.I. RosTOVTZEFF, Le classi rurali e le classi cittadine nell'Alto impero, in ID., Per la storia economica e sociale del mondo ellenistico e romano. Saggi scelti, a cura di T. Gnoli e J. Thornton, Catania 1995, 167 ss., nel leggere le riforme severiane nella chiave dell'antagonismo tra borghesia e contadini.

${ }^{53}$ M. FELICI, Profili storico-giuridici del pluralismo cittadino in Roma antica, Roma 2013, 83 ss.; in generale vedi anche G.A. CECCONI, La città e l'impero. Una storia del mondo romano dalle origini a Teodosio il Grande, Roma 2012, 285 ss.

54 «Poiché ci è congenito desiderare per tutto il nostro ecumene l'accrescimento del prestigio e del numero delle città e poiché vediamo che costoro [gli abitanti di Tymandus in Pisidia] desiderano nobilmente di ottenere il nome e la dignità di città, e dal momento che gli stessi assicurano che avranno una quantità di decurioni locali, abbiamo deciso di rispondere positivamente». 
Ad ogni modo, l'età felice dell'impero del II secolo d.C. - tale prevalentemente per le classi elevate, per i ricchi, per gli intellettuali cui veniva assicurata da sovrani tolleranti la libertà d'opinione ${ }^{55}$, mentre lo fu meno per le classi inferiori - era destinata a dissolversi presto. La fine traumatica dei Severi fece cadere la patina dorata che tanto fascino ha esercitato anche sui moderni, per far affiorare prepotentemente l'iceberg della degenerazione politica di un impero in corsa verso la profonda frattura tra ceti che si sarebbe presto consumata con tutta la sua portata deflagrante: corruzione, cinismo, sete di ricchezza delle classi dirigenti centrali e locali, privatizzazione delle strutture imperiali e del patrimonio pubblico, forti spinte corporative determinarono una divaricazione sociale e culturale sempre più ampia tra classi superiori e ceti popolari, e ancor più accentuata dalle masse rurali. La separatezza fu ulteriormente aggravata dallo sgretolamento della severità e della sobrietà, virtù secolari della nobiltà centrale alla testa di Roma e di quelle locali delle comunità cittadine, cagionato da poderose trasformazioni economiche e sociali.

Il grande latifondo, attestatosi nella villa, assunse progressivamente altre fattezze per delineare una nuova, diversa entità. Alla funzione principale di produzione economica della villa romana si affiancava quella della sicurezza e difesa del territorio declinato in un'ottica autarchica (o di autonomia) in stretto rapporto con il disimpegno politico dell'aristocrazia senatoria terriera (ripiegata sulla cura della rendita fondiaria e sempre più lontana dalla tradizionale ideologia del bene comune), quali aspetti peculiari di una embrionale morfologia feudale di un'incipiente economia curtense ${ }^{56}$. Tutti fenomeni in nuce ma rispetto ai quali nulla servì, se non forse soltanto a rinviare di poco la crisi, la concessione generale della cittadinanza romana con la constitutio antoniniana del 212 d.C., da cui restarono però escluse appunto le comunità non urbane e ingenti masse rurali ${ }^{57}$. E proprio qui stava il problema destinato ad aggravarsi. L'eredità augustea restava sul tappeto ma non più raccolta. E allora si cominciò a guardare all'impero in maniera diversa, non più come alla casa comune, non più al luogo dell'identità e della sicurezza, ma a scorgervi un'entità lontana, ostile, vessatoria, persino nemica. Un sentimento collettivo tanto più aggressivo quanto distante era la percezione del potere imperiale che, a differenza di quanto accadeva in Oriente, nei territori occidentali appunto andava frantumandosi.

${ }^{55}$ Così Nicola Palazzolo, in O. LiCANDRo - N. PALAZzolo, Roma e le sue istituzioni dalle origini a Giustiniano, Torino 2019, 237, che sottolinea come quel «secolo di pace [...] ebbe il suo contrappeso in un quasi totale immobilismo nella vita politica, sociale e culturale, che raggiunse l'acme durante il principato di Antonino Pio»).

${ }^{56}$ Sui profondi cambiamenti della Tarda Antichità si rinvia a P. ANDERSON, Dall 'antichità al feudalesimo, Milano 1978; AA.VV., Società romana e impero tardoantico. I. Istituzioni, ceti, economie, a cura di A. Giardina, Roma-Bari 1986; F. DE MARTINO, Uomini e terre in Occidente. Tra tardo antico e medioevo, Napoli 1988.

${ }^{57}$ Nonostante l'ingente letteratura, credo ancora meritevole di riflessione il tema attualissimo della cittadinanza e della sua concessione universale, su cui vedi il mio recente contributo Doppio domicilio e doppia cittadinanza. Strumenti di governo ed egemonia politica tra 'leges' e 'prudentes' nell'età repubblicana, in AUPA 61 (2018) 145 ss. (segmento di un itinerario di ricerca in corso). In generale, limitandomi alla bibliografia più recente, si legga T. SPAGNUOLO VIGORITA, Cittadini e sudditi tra II e III secolo, in AA.VV., Storia di Roma. III. L'età tardoantica. I. Crisi e trasformazioni, dir. A. Schiavone, Torino 1993, 5 ss.; ID., Città e impero. Un seminario sul pluralismo cittadino nell'impero romano, Napoli 1996; V. MAROTTA, La cittadinanza romana in età imperiale (secoli I-III d.C.). Una sintesi, Torino 2009, 101 ss.; ID., Tre riflessioni sulla cittadinanza: da Roma antica al mondo attuale, in IAH 5 (2013) 53 ss.; ID., Egizi e cittadinanza romana, in Cultura giuridica e diritto vivente 1 (2014) 1 ss.; ID., Doppia cittadinanza e pluralità degli ordinamenti cittadini, cit., 461 ss.; G. PURPURA, Constitutio Antoniniana de civitate, in AA.VV., Revisione ed integrazione dei Fontes Iuris Romani Anteiustiniani (FIRA). Studi preparatori. I. Leges (a cura di G. Purpura), Torino 2012, 695 ss.; ID., Il P. Giss. 40, I, in IAH 5 (2013) 73 ss.; A. TORRENT, La Constitutio Antoniniana. Reflexiones sobre el Papiro Giessen 40 I, Madrid 2012; C. CoRBO, Constitutio Antoniniana. Ius Philosophia Religio, Napoli 2013 ; A. MASTINO, Constitutio Antoniniana: la politica della cittadinanza di un imperatore africano, in BIDR 107 (2013) 37 ss.; A. PALMA, Note in tema di cittadinanza romana e sovranità, in Koinonia 38 (2014) 279 ss. 
In questo confuso e fluido contesto, quel grande corpo sociale fatto di decurioni, piccoli e medi contadini, artigiani, commercianti, militari (oggi ne parleremmo in termini di classe media) in quei territori di fondamentale importanza dell'impero e maggiormente aggrediti dalla crisi e dall'insicurezza sociale, si frammentava, si polverizzava e conseguentemente sbiadiva il senso di sé, della propria appartenenza all'impero, tanto da far credere, a un certo punto, del tutto disperso, smarrito il sentimento comune, quel sentimento collettivo di orgogliosa identità efficacemente sintetizzato da Erodiano per cui «Roma è dove si trova l'imperatore» (Herod. 1.6.5) ${ }^{58}$. Non tardarono così a scoppiare, sempre più frequentemente, rivolte come quella dei Bacaudae (contadini celtico-romani autori di rilevanti sollevazioni) in una terra importante e florida, comunque strategica per l'impero, come la Gallia.

Simili fenomeni insurrezionali però abbandonarono presto il loro tratto di episodicità e di valenza locale, per innestarsi in processi ancor più ampi avviati da gravi focolai di tendenze separatiste, non solo espressione di forme illegittime del potere e del suo esercizio, ma anche foriere di un fenomeno di disgregazione dell'impero.

Per avere un'idea della caotica frammentarietà del quadro politico e istituzionale basti ricordare uno dei casi tra i più eclatanti e interessanti dei secoli tardoantichi, denso di gravi pericoli per la vita dell'impero: l'usurpazione di Postumo e della costituzione, per quanto di breve durata, del suo singolare Imperium Galliarum. Il fenomeno secessionista, che aveva al proprio interno altri elementi peculiari appunto come i Bacaudae, coprì circa un quindicennio (260-274 d.C.) e, dopo Postumo, vide avvicendarsi, come figure altrettanto effimere di imperatori delle Gallie, dapprima Mario, poi Vittorino, e infine Tetrico. L'inquietante parentesi gallica costituì in effetti un vero pericolo per la tenuta dell'Occidente imperiale: per quanto di portata macroregionale, quell'esperienza secessionista interessò dapprima le Gallie ma presto si estese, contaminando Spagna e Britannia. A tutto ciò si aggiungeva la gestione della 'questione Barbari' - tra accoglienza (strumentale a fini di difesa militare) e contrasto, versanti ideologici lungo i quali si divideva profondamente l'opinione pubblica - che in larghi strati della popolazione dell'impero produceva sentimenti di ostilità per una percezione di insicurezza e pericolo da essa determinata. Insomma, l'impero sembrava davvero sul punto di cedere.

\section{CONCLUSIONE}

Oggi, nella formidabile e infuocata temperie mondiale che ci attraversa, con l'ansia tracimante, e spesso strumentale, di sicurezza, sollevata da retrive pulsioni nazionaliste definite da una suadente semantica espressioni di un'impellenza di 'populismo' o 'sovranismo' non è inutile continuare a riflettere, andando indietro nel tempo, e comprendere se e come analoghi problemi della pressante contemporaneità furono assunti e affrontati in quella straordinaria esperienza giuridica la cui eredità oggi è prezioso patrimonio della cultura europea. Non c'è nessun tentativo, cosa che apparirebbe quantomeno stravagante, di proporre la ricetta augustea ai problemi della nostra attualità, ma semmai sottolineare che i problemi fondamentali dell'uomo, per quanto possano mutare nella forma, restano gli stessi o assai simili e alla cui soluzione dovranno provvedere la politica e la scienza giuridica fino a quando saranno potenti e occuperanno un posto centrale nel governo e, più in generale, nell'esistenza umana. È questo piuttosto l'insegnamento da trarre dalle scienze storiche e dunque dalla storia del diritto, oggi sotto attacco, e da trasmettere alle nuove generazioni di studenti e studiosi, affermandone appunto la somma 'utilità' per le stesse

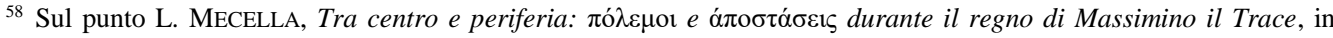
AA.VV., Erodiano tra crisi e trasformazioni (a cura di A. Galimberti), Roma 2017, 187 ss.
} 
scienze giuridiche in generale, anch'esse oggi messe a repentaglio da quella che non può che definirsi un'autentica 'età di ferro' dell'ideologia tecnocratica e tecnologica.

In conclusione: sovranità popolare, legge, valori etici, selezione della classe dirigente su merito e virtù, saperi, diritto, identità e cittadinanza, strumenti di governo, pluralismo normativo, furono tutti ingredienti della 'vicenda' politica e istituzionale dell'esperienza statale e giuridica di Roma; una lezione da cui possiamo ancora oggi utili trarre indicazioni ma di cui, purtroppo, la nostra Vecchia Europa rischia di perdere la memoria. E purtuttavia, proprio in tempi di crisi formidabili, che recano in sé anche una crisi, forse irreversibile, del modello occidentale di democrazia, appare quasi come un conforto, se non un auspicio o fors'anche un relitto a cui aggrapparsi, una stilla pura di pensiero del Geist di Rudolph von Jhering: «Nessuna cosa veramente grande perisce in questo mondo» ${ }^{59}$.

\section{RIFERIMENTI}

AA.VV., Ovidio. Amori, miti e altre storie, Roma 2018.

AA.VV., Società romana e impero tardoantico. I. Istituzioni, ceti, economie, a cura di A. Giardina, Roma-Bari 1986.

P. ANDERSON, Dall'antichità al feudalesimo, Milano 1978.

A. Bancalari Molina, Orbe Romano e Imperio Global. La Romanización desde Augusto to Caracalla, Santiago, 2007.

S. BARBATI, Gli studi sulla cittadinanza romana prima e dopo le ricerche di Giorgio Luraschi, 175 ss.

A. BARICCO, The Game, Torino 2018.

A. BARICCO, The Game Unplugged, Torino 2019.

O. BEHRENDS, Die Rechtsregeln der Militärdiplome und das die Soldaten des Prinzipats treffende Eheverbot, in AA.VV., Heer und Integrationspolitik (hrsg. W. Eck, H. Wolff), KölnWien 1986, 123 ss.

P. Buongiorno, Ateii Capitones, in Iura 59 (2011) 195 ss.

P. Buongiorno, Senatus consulta Claudianus temporibus facta. Una palingenesi delle deliberazioni senatorie dell'età di Claudio (41-54 d.C.), Napoli 2010.

P. Buongiorno, C. Ateius Capito. Appunti per una biografia politica, in Scritti per A. Corbino (a cura di I. Piro), I, Tricase (LE) 2016, 413 ss.

L. CApogrossi Colognesi, A Provocation, in Rivista di Storia Economica 25 (2009) 421 ss.

L. CApogrossi Colognesi, La genesi dell'impero municipale, in Roma e America. Diritto romano comune 18 (2004) 243 ss.

L. CAPOgRossi Colognesi, Padroni e contadini nell'Italia repubblicana, Roma 2012.

F.P. CASAVOLA, Il concetto di "Urbs Roma”; giuristi e imperatori romani, in Labeo 38 (1992) $20 \mathrm{ss}$.

${ }^{59}$ R. VON JHERING, Geist des römischen Rechts auf den verschiedenen Stufen seiner Entwicklung, I, Leipzig $1907,13$. 
G.A. CECCONI, La città e l'impero. Una storia del mondo romano dalle origini a Teodosio il Grande, Roma 2012.

P. CERAMI - M. Miceli, Storicità del diritto. Strutture costituzionali, Fonti, Codici. Prospettive romane e moderne, Torino 2018, 64 ss.

P. CERAMI - A. PETRUCCI, Diritto commerciale romano. Profilo storico, Torino 2010.

C. Corbo, Constitutio Antoniniana. Ius Philosophia Religio, Napoli 2013.

O.D. CORDOVAnA, I Severi, in AA.VV., Roma universalis. L'impero e la dinastia venuta dall'Africa, Milano 2018, 36 (catalogo della mostra).

O.D. CoRdovanA, Segni e immagini del potere tra antico e tardoantico. I Severi e la provincia Africa proconsularis, Catania 2007.

F. DE MARTINO, Storia economica di Roma antica, II, Firenze 1979.

F. DE MARTINO, Uomini e terre in Occidente. Tra tardo antico e medioevo, Napoli 1988.

Elio Aristide, A Roma (traduzione e commento a cura di F. Fontanella, introduzione di P. Desideri), Pisa 2007.

PH. FABIA, La Table Claudienne de Lyon, Lyon 1929.

M. FELICI, Profili storico-giuridici del pluralismo cittadino in Roma antica, Roma 2013.

R. Frei-Stolba, H. LiEB, Un diplôme civil: le fragment de Carnuntum, in ZPE 143 [2003] 243 ss.

F. FUKUYAMA, Identità. La ricerca della dignità e i nuovi populismi, Milano 2019.

L. GAGLIARDI, Osservazioni in tema di domicilio degli incolae. La distinzione tra incolae di città e incolae di campagna, in AA.VV., Gli Statuti Municipali (a cura di L. Capogrossi Colognesi, E. Gabba), Pavia 2006, 647 ss.

E. GABBA, Dallo stato-città allo stato municipale, in AA.VV., Storia di Roma. 2. L'impero mediterraneo. I. La repubblica imperiale (dir. A. Schiavone), Torino 1990, 697 ss.

E. GABBA, I municipi e l'Italia augustea, in AA.VV., Continuità e trasformazioni fra repubblica e impero. Istituzioni, politica, società, Bari 1991, 69-82.

E. GaBBA, La rifondazione di Salapia, in Athenaeum 71 (1983) 514 ss.

E. GABBA, Le città italiche del I sec. a.C. e la politica, in RSI 98 (1986) 653 ss.

E. GABBA, L'impero di Augusto, in AA.VV., Storia di Roma. 2. L'impero mediterraneo. II. I principi e il mondo (dir. A. Schiavone), Torino 1991, $26 \mathrm{s.}$

E. GabBA, Municipium Augustum Veiens, in Athenaeum 86 (1988) 203 s.

F GHEDINI, Il poeta del mito. Ovidio e il suo tempo, Roma 2018.

C. GIACHI, La Tabula Banasitana: cittadini e cittadinanza ai confini dell'Impero, in AA.VV., Civis/Civitas, Cittadinanza politico-istituzionale e identità socio-culturale da Roma alla prima età moderna. Atti del Seminario internazionale di Siena-Montepulciano, 10-13 luglio 2008 (a cura di C. Tristano, S. Allegria), Montepulciano (SI) 2009, 17 ss.

V. GIUFFRÈ, La struttura politico-costituzionale e il territorio, in AA.VV., Città territorio e diritto privato nei primi due secoli dell'impero. Atti del convegno internazionale di diritto romano, Copanello 5-8 giugno 2002 (a cura di F. Milazzo), Soveria Mannelli 2010, 26. 
CH. GuILluy, La società non esiste. La fine della classe media occidentale, Roma 2019.

R. HINGLEY, Globalizing Roman Culture. Diversity and Empire, London-New York 2005.

TH. HYLLAND ERIKSEN, Fuori controllo. Un'antropologia del cambiamento accelerato, Torino 2017.

N. IRTI, Norma e luoghi. Problemi di geo-diritto, Roma-Bari 2002.

R.B. Hitchner, The First Globalization. The Roman Empire and Its Legacy in the 21st Century, Oxford 2007.

U. LAFFI, Studi di storia romana e di diritto, Roma 2001.

U. LAFFI, Colonie e municipi nello Stato romano, Roma 2007.

F. LAMBERTI, «Tabulae Irnitanae». «Municipalità» e «Ius Romanorum», Napoli 1993.

O. LATTIMORE, La frontiera. Popoli ed imperialismi alla frontiera tra Cina e Russia, Torino 1970.

A.S. LEWIN, Popoli, terre, frontiere dell'Impero romano. Il vicino Oriente nella tarda antichità. I: Il problema militare, Catania 2008.

O. LiCANDRo, Augusto e la res publica imperiale. Studi epigrafici e papirologici, Torino 2018.

O. LICANDRO, Cicerone alla corte di Giustiniano. "Dialogo sulla scienza politica" (Vat. gr. 1298). Concezioni e dibattito sulle formae rei publicae nell'età dell'assolutismo imperiale, Roma, 2017.

O LICANDRO, Dalla lex Claudia de quaestu senatorum alle leges repetundarum ovvero del conflitto di interessi nell'antica Roma, in Fides Humanitas Ius. Studii in onore di L. Labruna, IV, Napoli 2007, 2815 ss.

O. LICANDRO, Domicilium e incolae tra repubblica e principato, in AA.VV., Étrangers dans la cité romaine. "“Habiter une patrie”: des incolae de la République aux peuples fédérés du BasEmpire». Actes du Colloque de Valenciennes (14-15 octobre 2005), Rennes 2007, 66 ss.

O LICANDRO, Doppio domicilio e doppia cittadinanza. Strumenti di governo ed egemonia politica tra 'leges' e 'prudentes' nell'età repubblicana, in AUPA 61 (2018) 145 ss.

O LiCANDRO, Pomponio e L'incola. Osservazioni su D. 50.16.239.2 (Pomp. 1. sing. ench.) alla luce di Lex Urs. cap. 98 e Lex Irn. cap. 83, in fil...a. Scritti per Gennaro Franciosi, a cura di F.M. D’Ippolito, II, Napoli 2007, 1381 ss.

O. LICANDRO - N. PALAZZOLO, Roma e le sue istituzioni dalle origini a Giustiniano, Torino 2019.

E. Lo CASCIO, Forme dell'economia imperiale, in AA.VV., Storia di Roma. 2. L'impero mediterraneo. II. I principi e il mondo (dir. A. Schiavone), Torino 1991, 358 ss.

E. LO CASCIO, La "New Institutional Economics" e l'economia imperiale romana, in AA.VV., Storia romana e storia moderna. Fasi in prospettiva (a cura di M. Pani), Bari 2005, 313 ss.

E. LO CASCIO, L'economia imperiale e la svolta augustea, in AA.VV., Augusto e la costruzione del principato. Atti del Convegno (Roma, 4-5 dicembre 2014), Roma 2017, 327 ss. 
E. LO CASCIO, Roma come «mercato comune del genere umano», in AA.VV., Elio Aristide e la legittimazione greca dell'impero di Roma, a cura di P. Desideri e F. Fontanella, Bologna 2013, 193.

E. Lo CASCIO, The Early Roman Empire: the State and the Economy, in AA.VV., The Cambridge Economic History of the Greco-Roman World (ed. W. Scheidel, I. Morris, R. Saller), Cambridge 2007, 619 ss.

G. LuRASCHI, Foedus Ius Latii Civitas. Aspetti costituzionali della romanizzazione in Transpadania, Padova 1979.

V. MAROTTA, Doppia cittadinanza e pluralità degli ordinamenti cittadini. La Tabula Banasitana e le linee 7-9 del Papiro di Giessen 40 col. I, in AG 236 [2016] 461 s.

V. MAROTTA, Egizi e cittadinanza romana, in Cultura giuridica e diritto vivente 1 (2014) 1 ss. V. MAROTTA, La cittadinanza romana in età imperiale (secoli I-III d.C.). Una sintesi, Torino 2009.

V. MAROTTA, Tre riflessioni sulla cittadinanza: da Roma antica al mondo attuale, in IAH 5 (2013) 53 ss.;

A. MASTINO, Constitutio Antoniniana: la politica della cittadinanza di un imperatore africano, in BIDR 107 (2013) 37 ss.

M. MAZZA, Identità etniche e culture locali sulla frontiera dell'Eufrate (II-IV sec. d.C.). Uno studio sui contatti culturali, in ID., Cultura guerra e diplomazia nella Tarda Antichità. Tre studi, Catania 2005, 13 ss.

S. MAZZARINO, L'Impero romano, II, Roma-Bari 1988.

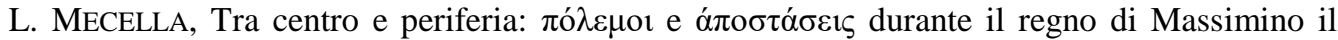
Trace, in AA.VV., Erodiano tra crisi e trasformazioni (a cura di A. Galimberti), Roma 2017, 187 ss.

M. MICELI, 'Governo misto', quartum genus rei publicae e separazione dei poteri, in AA.VV., Tradizione romanistica e Costituzione (dir. L. Labruna - a cura di M.P. Baccari e C. Cascione), I.1, Napoli 2006, 659 ss.

E. MiglaRIO, Gentes foederatae. Per una riconsiderazione dei rapporti romano-berberi in Mauretania Tingitana, in RAL 10 (1999) 427 ss.

C. NICOLET, L'inventario del mondo. Geografia e politica alle origini dell'impero romano, Roma-Bari 1989.

A. PALMA, Note in tema di cittadinanza romana e sovranità, in Koinonia 38 (2014) 279 ss.

G. POMA, Nota su CIL, VIII, 9228: populis novis ex Africa inlatis, in AA.VV., Roma e i 'diversi'. Confini geografici, barriere culturali, distinzioni di genere nelle fonti letterarie ed epigrafiche fra età repubblicana e Tarda Antichità, a cura di C. Giuffrida, M. Cassia e G. Arena, Milano 2018, 26 ss.

G. PURPURA, Constitutio Antoniniana de civitate, in AA.VV., Revisione ed integrazione dei Fontes Iuris Romani Anteiustiniani (FIRA). Studi preparatori. I. Leges (a cura di G. Purpura), Torino 2012, 695 ss. 
G. PURPURA, Edicta Augusti ad Cyrenenses (6/4 a.C.), in AA.VV., Revisione ed integrazione dei Fontes Iuris Romani Anteiustiniani (FIRA). Studi preparatori. I. Leges (a cura di G. Purpura), Torino 2012, 433 ss.

G. PURPURA, Il P. Giss. 40, I, in IAH 5 (2013) 73 ss.

A. RAGGI, Seleuco di Rhosos. Cittadinanza e privilegi nell'Oriente greco in età tardo-repubblicana, Pisa 2006.

S. RODA, Imperium sine fine e i confini dell'Impero tra ideologia, propaganda e Realpolitik, in AA.VV., Roma e i 'diversi'. Confini geografici, barriere culturali, distinzioni di genere nelle fonti letterarie ed epigrafiche fra età repubblicana e Tarda Antichità, a cura di C. Giuffrida, M. Cassia e G. Arena, Milano 2018, 73 ss.

S. RonCATI, Caio Ateio Capitone e i Coniectanea (Studi su Capitone, I), in SDHI 71 (2005) 271 ss.

M.I. RostovtzefF, Le classi rurali e le classi cittadine nell'Alto impero, in ID., Per la storia economica e sociale del mondo ellenistico e romano. Saggi scelti, a cura di T. Gnoli e J. Thornton, Catania 1995, 167 ss.

M. RostovZEV, Storia economica e sociale dell'impero romano, Firenze 1980.

F. RUSSO, L'odium regni a Roma tra realtà politica e finzione storiografica, Pisa, 2015.

P. SAGE, La Table claudienne et le style de l'empereur Claude: essai de réhabilitation, in REL 58 (1980) 274 ss.

A. SCHIAVOne, La storia spezzata. Roma antica e Occidente moderno, Roma-Bari 2002.

C. SCHMITT, Il nomos della terra nel diritto internazionale dello «jus publicum europaeum», Milano 1991.

T. SPAGNUOlO VIGORITA, Città e impero. Un seminario sul pluralismo cittadino nell'impero romano, Napoli 1996.

T. Spagnuolo Vigorita, Cittadini e sudditi tra II e III secolo, in AA.VV., Storia di Roma. III. L'età tardoantica. I. Crisi e trasformazioni, dir. A. Schiavone, Torino 1993.

E. STOLFI, 'Plurima innovare instituit'. Spunti esegetici intorno al confronto tra Labeone e Capitone in D. 1.2.2.47 (Pomp lib. Sing. ench.), in Studi per G. Nicosia, VIII, Milano 2007, 57 ss.

A. TORRent, La Constitutio Antoniniana. Reflexiones sobre el Papiro Giessen 40 I, Madrid 2012.

R. TURCAN, Claude de Lyon: un précurseur, in BIDR 107 (2013) 21 ss.

F.J. TURNER, La frontiera nella storia americana, Bologna 1975.

O. VEYNE, L’Impero greco romano. Le radici del mondo globale, Milano 2007.

E. Volterra, La Tabula Banasitana. A proposito di una recente pubblicazione, in BIDR 77 (1974) 407 ss.

R. VON JHERING, Geist des römischen Rechts auf den verschiedenen Stufen seiner Entwicklung, I, Leipzig 1907.

J.B. WARD-PERKINS, Frontiere politiche e frontiere culturali, in AA.VV., La Persia e il mondo romano, Accademia dei Lincei, Quaderno 76, Roma 1966, 395 ss.. 
M. WEBER, Economia e società. L'economia in rapporto agli ordinamenti e alle forze sociali. La città (a cura di W. Nippel), Roma 2003.

G. ZeCCHINI, Polibio. La solitudine dello storico, Roma 2018.

F. ZuCCOTTI, Sacramentum civitatis. Diritto costituzionale e ius sacrum nell'arcaico ordinamento giuridico romano, Milano 2016.

Recebido em: 16 mar. 2020.

Aceito em: 28 abr. 2020. 